To appear in Vehicle System Dynamics

Vol. 00, No. 00, Month 20XX, 1-27

\title{
Motion Cueing in High-Performance Vehicle Simulators
}

\author{
I.G. Salisbury ${ }^{\mathrm{*} *}$ and D.J.N. Limebeer ${ }^{\mathrm{a}}$ \\ ${ }^{a}$ Department of Engineering Science, University of Oxford, Oxford, UK
}

(Received 00 Month 20XX; accepted 00 Month 20XX)

\begin{abstract}
The majority of motion cueing algorithms have been developed for passenger car applications, with correspondingly less research dedicated to race-car and high-performance vehicle simulators. In the high-performance context, the focus is on cueing the vehicle's behavioural and handling characteristics, particularly when driving near the limits of performance. In race car simulators the cueing requirements are therefore quite different, with the problem made all the more challenging by the presence of large accelerations. To understand the drivers' cueing needs, the vehicle's stability and handling response characteristics must be examined near the performance boundary. Frozen-time eigenvalue analyses are used to determine stability and response characteristics across all vehicle operating conditions, including accelerating and braking under cornering, with the results used to determine motion cueing algorithm requirements. Lateral acceleration and yaw cueing filters are designed in order to retain information critical to understanding the vehicle's behaviour on its performance boundary. Cueing filters are then tested, with the help of a professional race car driver, and are found to provide the cues necessary for the driver to control the vehicle on the limit of performance.
\end{abstract}

Keywords: high-performance driving simulator; motion cueing; vehicle handling and stability;

\section{Introduction}

Driving simulators can be subdivided into various classes depending on the application. One group of simulators is used to study human behaviour, including the interaction of the driver and vehicle assistance systems, and the driver's response to critical situations. These applications envisage the use of passenger vehicles in highway and city driving. A second class of simulators is used to study vehicular behaviour and will typically involve a professional test driver evaluating the vehicle on its performance limit in order to determine the ease with which it can be controlled and operated. This type of study is particularly important for the design of high-performance road and race cars.

In both cases high-fidelity simulators consist of advanced graphics systems, a realistic cockpit with actuated controls and a moving platform. The dynamic motion base provides acceleration cues that stimulate the driver's vestibular system (biological accelerometers). These signals improve the drivers' perception of the vehicle's behaviour, and assist the driver, for example, to brake at more realistic rates [1].

In broad terms dynamic driving simulators aim to reproduce vehicular accelerations [2]. In order to satisfy workspace and bandwidth constraints, vehicular acceleration signals

\footnotetext{
*Corresponding author. Email: ingrid.salisbury@eng.ox.ac.uk
} 
must be filtered and/or attenuated and consequently only part of the vehicle's movement is rendered by the motion platform. Motion cueing algorithms have evolved to address the design of these filters [3]. Most of the approaches described in the literature aim to minimise the difference between the accelerations perceived in the simulator and those perceived in the real vehicle, where the perception model is a linear transfer function representing the vestibular dynamics [4].

This approach is successful in passenger car simulators that only respond to lowmagnitude accelerations and slow dynamics. Sustained longitudinal and lateral accelerations that cannot be reproduced are substituted with pitch and roll motions in a technique called tilt 'co-ordination'. Tilt co-ordination relies on the vestibular system 'misconstruing' the components of the acceleration due to gravity in the longitudinal and lateral directions as translational accelerations rather than tilt. With these methods, it is possible to reproduce many features of the sensed accelerations. However, in race-car and high-performance vehicle applications, the vehicle accelerations are an order of magnitude larger than those in typical passenger cars, and so only a small fraction of the motion is rendered. Since the perceptual error is large relative to the simulator motion, the incorporation of a vestibular model does not offer much assistance in the cueing algorithm design. Furthermore, fast dynamics make tilt co-ordination ineffective.

There is little value in attempting to replicate vehicular accelerations, since it is both unachievable and unnecessary. Experienced test drivers do not need to 'believe' the simulator is a car. They merely require sufficient information to be able to control and drive the car realistically. The cueing focus should therefore be on providing the correct sensory inputs and minimise the difference between the driver's control behaviour in the simulator and a real vehicle.

In racing, simulators are used to evaluate the set-up and its effect on the vehicle handling. To perform this task the driver needs sensory information from which he/she can infer the vehicle behaviour at the performance limit. This behaviour is influenced by the vehicle's handling and stability characteristics. By studying the changes in the car's stability and response characteristics as it approaches the limit, the requisite motion cues for the vehicle simulator can be analysed. This insight can be used to evaluate existing and develop new cueing methodologies.

Since the stability of the vehicle changes with speed and acceleration (both longitudinal and lateral), it is convenient to use a G-G diagram (Figure 1) to characterise the operating domain of the car. This well-known diagram, plots the lateral vehicle acceleration against the longitudinal acceleration with a boundary line representing the physical limits of the car. The engine power is the primary influence on the positive longitudinal acceleration limit, while the tire grip is the key factor in determining the lateral and braking limits. A graphical overlay of recorded telemetry data, can be used to analyse the drivers' exploitation of the performance envelope. In racing, the more the driver is able to utilise the capability of the vehicle, the lower the lap time.

Handling and stability characteristics influence the drivers' ability to operate the vehicle on the limit of performance. An unstable vehicle may undermine the drivers' confidence, or in extreme cases make the vehicle undriveable. The highlighted regions in Figure 1 correspond to areas where the vehicle is typically unstable and more difficult to control, thus requiring highly skilled drivers. Characterising the vehicle stability across the G-G diagram is not only useful for understanding motion cueing requirements, but can also assist in predicting the extent to which the driver will be able to exploit the vehicle's performance.

The treatment of stability over the full operating range of the vehicle has produced a number of analysis techniques, but all have their limitations. Milliken [5] describes the 


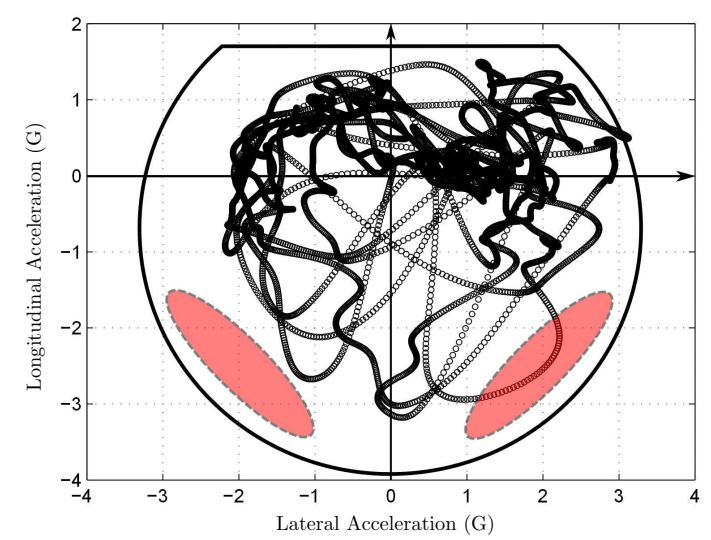

Figure 1. Sample G-G diagram of a Formula One car that shows the vehicle's lateral and longitudinal acceleration on a qualifying lap at Silverstone. The track contains 18 corners, of which 10 are right-handed, that accounts for the vehicle's positive-dominated lateral acceleration. In addition, the high-speed corners, out of which the driver accelerates, are also right-handed, which is consistent with vehicle's prolonged operation in the top-right quadrant of the diagram.

use of stability and control derivatives to assist in the assessment of vehicle handling such as oversteer and understeer [6]. These derivatives are generated from linearised vehicle models at particular steady-state operating points. Used originally in the aircraft industry, this approach is practically useful, and characterises the vehicle response to physical stimuli, such as an applied yaw moment. Despite providing valuable insight into vehicle behaviour, stability derivatives are only applicable to the portion of the G-G envelope corresponding to steady-state cornering conditions. More sophisticated methods use phase plane and bifurcation analysis to determine vehicle stability [7]. These techniques are only usable with relatively simple vehicle models.

This work uses Lyapunov's indirect method to study the stability of a vehicle under steady-state cornering conditions. The analysis is extended to accelerating and braking scenarios following the approach developed for motorcycle stability analysis [8] and more recently applied to the case of a racing vehicle G-G envelope in [9]. In essence, the car's stability properties are inferred from the eigenvalue of linearised vehicle models. While high-fidelity models can be used, due care must be taken with this type of analysis since it is only valid when the nonlinear system is 'varying slowly' [10, 11].

The time-varying eigenvalue analysis is first developed for accelerating and braking under straight running conditions. A simple two degree-of-freedom (2-DOF) car model facilitates the derivation of simple analytic expressions for the vehicle's stability and response. The stability analysis is then extended to the remainder of the operating chart by considering, first steady-state, and then accelerating and braking under cornering. A 3-DOF model is used that includes nonlinear, combined slip 'magic formula' type tires.

The changes in the vehicle stability and response under different operating conditions are analysed to determine the motion cues that are required by race-car simulator drivers. This insight is then utilised when tuning the lateral and yaw motion cueing algorithms. Finally, these algorithms are tested by a professional race-driver to assess the ease with which they allow the driver to control and drive the vehicle on its performance limit.

The remainder of this paper is arranged as follows: the analysis of stability under straight-running conditions is presented in Section 2. The extension to cornering scenarios is given in Section 3. Vehicular motion cueing as it approaches the limit of stability is discussed in Section 4. Cueing algorithm design is presented in Section 5, with tuning and 


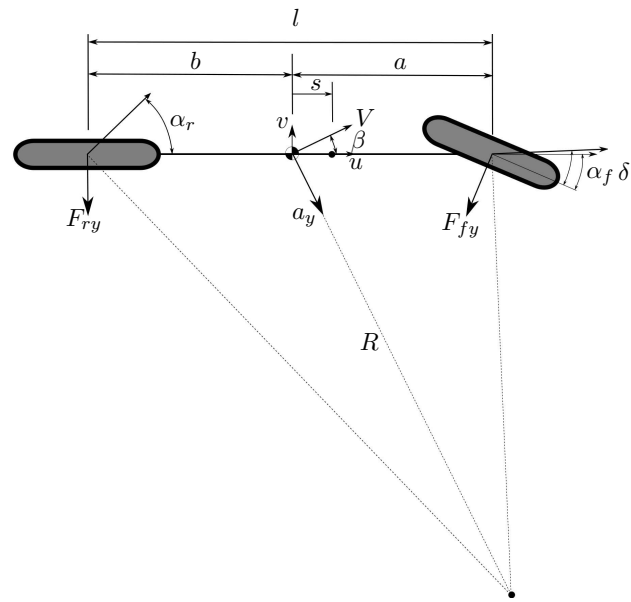

Figure 2. Kinematics of a single-track vehicle model showing its basic geometric parameters.

testing outcomes presented in Section 6. The work is concluded in Section 7.

\section{Straight Line Motion}

The stability of the straight-running car is studied first, with analytic results derived using a 2-DOF vehicle model.

\subsection{Vehicle Model}

A single-track bicycle model is used to describe the vehicle's dynamics and stability. The kinematics of the car model are shown in Figure 2, with all quantities described in an SAE coordinate system. Balancing forces in the longitudinal and lateral directions, and balancing yaw moments around the car's mass centre gives the following well known equations of motion (EOM):

$$
\begin{aligned}
m \dot{u} & =m \omega v-F_{f y} \sin \delta+F_{r x} \\
m \dot{v} & =-m \omega u+F_{f y} \cos \delta+F_{r y} \\
J \dot{\omega} & =a \cos \delta F_{f y}-b F_{r y} .
\end{aligned}
$$

The state vector is $x=[u, v, \omega]^{T}$, where the states are, respectively, the body-fixed longitudinal, lateral and yaw velocities. The parameters $a, b, m$ and $J$ represent respectively the distance of the front and rear axle from the centre-of-mass, the mass of the vehicle and the vehicle's yaw moment of inertia. The front wheel has steering angle $\delta$, which is assumed 'small'. The front and rear lateral tire forces are given by $F_{f y}$ and $F_{r y}$ respectively. The driven rear wheel has longitudinal tire force $F_{r x}$. The side-slip angle $\beta$ lies between the vehicle mass centre velocity $V$ and the vehicle's longitudinal axis. The front and rear tire velocities make slip angles $\alpha_{f}$ and $\alpha_{r}$ with the wheel plane directions. 
These three angles are computed using:

$$
\begin{aligned}
\beta & =\arctan \left(\frac{v}{u}\right), \\
\alpha_{f} & =\arctan \left(\frac{u \sin \delta-\cos \delta(\omega a+v)}{u \cos \delta+\sin \delta(\omega a+v)}\right), \\
\alpha_{r} & =\arctan \left(\frac{\omega b-v}{u}\right) .
\end{aligned}
$$

When the vehicle is accelerated, or braked, there is a normal tire force transfer between the front and rear axles. By balancing moments around the rear-wheel ground contact point, and noting that the sum of the tire down-forces is equal to the weight of the vehicle, the front and rear down-force, $F_{f z}$ and $F_{r z}$ respectively, can be computed as:

$$
\begin{aligned}
& F_{r z}=m g \frac{a}{a+b}+m a_{x} \frac{h}{a+b} \\
& F_{f z}=m g-F_{r z},
\end{aligned}
$$

where $h$ is the height of the centre-of-mass and $a_{x}$ is the longitudinal acceleration.

The lateral forces generated by the tires are assumed to depend linearly on the tire normal loads and slip angles, and are computed by:

$$
\begin{aligned}
& F_{f y}=C_{f 0} F_{f z}\left(\delta-\frac{\omega a+v}{u}\right), \\
& F_{r y}=C_{r 0} F_{r z}\left(\frac{\omega b-v}{u}\right),
\end{aligned}
$$

where, $C_{f 0}$ and $C_{r 0}$ are the normalised cornering stiffnesses (at the front and rear axle); (5) and (6) are simplified using small angle approximations.

Under straight-running conditions the influence of the $m w v$ term on the longitudinal acceleration is assumed negligible, which decouples the longitudinal speed from the other two EOM. We will assume that

$$
u(t)=u_{0}+a_{x} t .
$$

The tire model is combined with the remaining EOM, and the resulting linear timevarying EOM are given by:

$$
\begin{aligned}
& \dot{v}=-\frac{C}{m u(t)} v+\left(\frac{-C s}{m u(t)}-u(t)\right) \omega+\frac{C_{f}}{m} \delta, \\
& \dot{\omega}=\frac{-C s}{m k^{2} u(t)} v-\frac{C q^{2}}{m k^{2} u(t)} \omega+\frac{C_{f} a}{m} \delta,
\end{aligned}
$$


in which

$$
\begin{aligned}
C_{r} & =C_{r 0} F_{r z} \\
C_{f} & =C_{f 0} F_{f z} \\
C s & =C_{f} a-C_{r} b \\
C & =C_{f}+C_{r} \\
C q^{2} & =C_{f} a^{2}+C_{r} b^{2} \\
m k^{2} & =J .
\end{aligned}
$$

Following [12], the parameter ' $s$ ' is the distance between the centre-of-mass and the neutral steer point, $q$ is the average moment arm and $k$ the radius of gyration. The neutral steer point, which is shown in Figure 2, has kinematic significance; a force applied through this point will cause the vehicle to move laterally without yawing.

\subsection{Constant Velocity Stability}

Under steady-state conditions (12) and (13) are time-invariant. When examining the behaviour of the car, the response in the lateral acceleration, yaw velocity and side-slip angle to perturbations in steering angle are determined. These quantities are given by: $y=\left[a_{y}, \omega, \beta\right]^{T}$, where, the lateral acceleration is given by:

$$
a_{y}=\dot{v}+u \omega
$$

$\omega$ is a state, and $\beta$ is given in (4), but linearised assuming small angles.

The state-space model of the car from the steering angle to the output vector is given by:

$$
\begin{gathered}
A=\left(\begin{array}{cc}
-\frac{C}{m u_{0}} & \frac{-C s-m u_{0}^{2}}{m u_{0}} \\
\frac{-C s}{J u_{0}} & -\frac{C q^{2}}{J u_{0}}
\end{array}\right) B=\left(\begin{array}{c}
\frac{C_{f}}{m} \\
\frac{a C_{f}}{J}
\end{array}\right) \\
C=\left(\begin{array}{cc}
-\frac{C}{m u_{0}} & -\frac{C s}{m u_{0}} \\
0 & 1 \\
\frac{1}{u_{0}} & 0
\end{array}\right) \quad D=\left(\begin{array}{c}
\frac{C_{f}}{m} \\
0 \\
0
\end{array}\right)
\end{gathered}
$$

where $u_{0}$ is the steady-state velocity of the vehicle.

The stability of this constant-velocity model has been studied extensively, e.g. [12]. The poles of the system, which are used to determine the stability are the roots of the characteristic polynomial:

$$
\lambda^{2}+\frac{\left(a^{2}+k^{2}\right) C_{f}+\left(b^{2}+k^{2}\right) C_{r}}{m k^{2} u_{0}} \lambda+\left(\frac{l^{2} C_{f} C_{r}}{m^{2} k^{2} u_{0}^{2}}-\frac{C s}{m k^{2}}\right)=0 .
$$

Following the Routh-Hurwitz criterion, the system will be stable if all the coefficients in (22) are positive. With a linear tire model all cornering stiffnesses are positive, hence only the last term in (22) can become negative, which occurs when the speed exceeds 
some critical value given by:

$$
u_{0}>V_{\text {crit }}=\sqrt{\frac{l^{2} C_{f} C_{r}}{m C s}} .
$$

The existence of a positive, real critical speed is dictated by the sign of the $C s$ term. Recalling equation (16), this depends on the location of the centre-of-mass and the cornering stiffnesses of the tires. This is linked to the concepts of over and understeer, which have a number of equivalent definitions $[5,12]$. The definition given here is not restricted to a linearised tire model, and is chosen because it links the ideas to the driver input. If a vehicle is negotiating a turn of constant radius, and the speed is increased, the steering angle required to maintain the turn will change. In an under-steering vehicle it will increase, in an oversteering vehicle it will decrease and in a neutrally steering vehicle it will remain constant. It can be shown that if $C s$ is positive, the vehicle will oversteer and hence have a critical speed above which it is unstable [12]. If, on the other hand, it is negative, the vehicle will under-steer and will remain stable at all speeds.

The stability and steering characteristics of a race car will now be studied, with the vehicle parameters given in Table A1 in the Appendix. The tire parameters are chosen in order to give an oversteering vehicle with a critical speed of $V_{\text {crit }}=65 \mathrm{~m} / \mathrm{s}$.

From the state-space model in (21) the transfer functions from the steering angle to each of the outputs are determined. While the system poles are determined by the characteristic equation, the zeros of each of the three transfer functions are given by

$$
\begin{aligned}
\left\{z_{a_{y} 1}, z_{a_{y} 2}\right\} & =-\frac{b C_{2} l}{2 J u_{0}} \pm \sqrt{\left(\frac{b C_{2} l}{2 J u_{0}}\right)^{2}-\frac{C_{2} l}{J}} \\
z_{\beta} & =\frac{a m u_{0}^{2}-b C_{2} l}{J u_{0}} \\
z_{\omega} & =-\frac{C_{2} l}{m u_{0} a}
\end{aligned}
$$

The zeros of the lateral acceleration transfer function will either be negative real, or will form a complex conjugate pair with a negative real part. The zero of the yaw velocity response transfer function will always be negative, however, at higher speeds, the zero of the side-slip angle transfer function may become positive, resulting in a non-minimum phase response.

The system poles and the zeros of each transfer function are given in the left-half of Figure 3 for vehicle speeds from $20 \mathrm{~m} / \mathrm{s}$ to $80 \mathrm{~m} / \mathrm{s}$. As the speed increases, the poles of the system migrate towards the right-half plane, with one becoming positive when the critical speed of $65 \mathrm{~m} / \mathrm{s}$ is reached.

The lateral acceleration, side-slip angle and yaw velocity responses to a step change in the steering angle are determined at speeds of $u_{0}=\{30 \mathrm{~m} / \mathrm{s}, 50 \mathrm{~m} / \mathrm{s}, 70 \mathrm{~m} / \mathrm{s}\}$. The results are shown in the right half of Figure 3 . At $\mathrm{u}_{0}=30 \mathrm{~m} / \mathrm{s}$ the system responses exhibit overdamped, stable behaviour, approaching finite final values. At $u_{0}=50 \mathrm{~m} / \mathrm{s}$ the responses are quicker, with the outputs achieving significantly larger values in the same time. At $\mathrm{u}_{0}=70 \mathrm{~m} / \mathrm{s}$ the system is unstable and the responses grow indefinitely. The non-minimum phase zero of the side-slip angle transfer function produces an initial movement in the opposite direction in the response, which differentiates it from the yaw behaviour. 

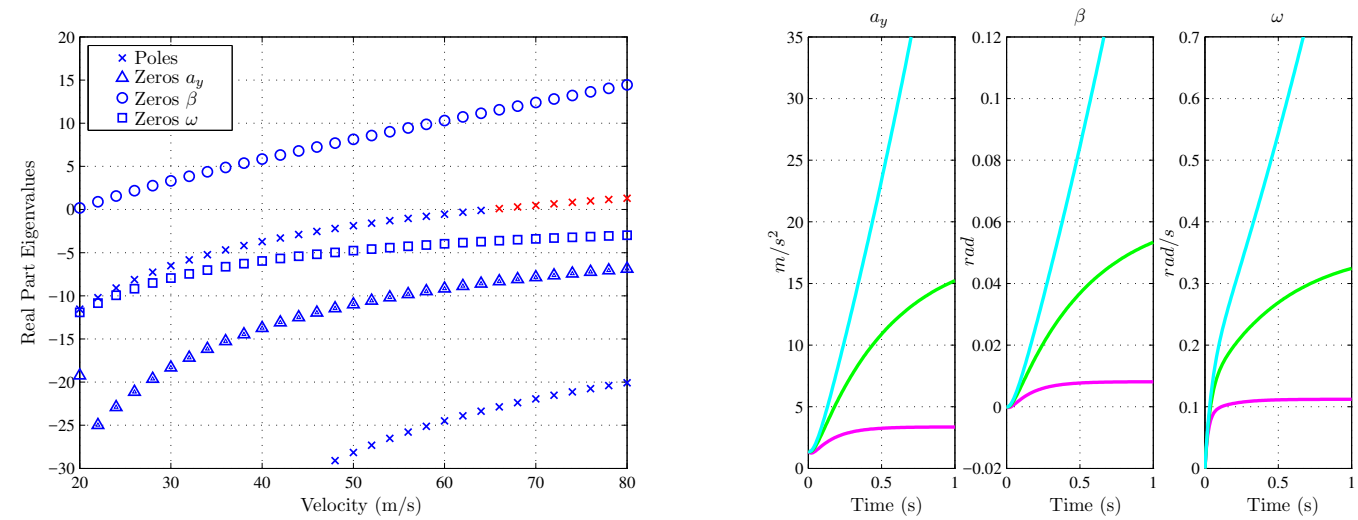

Figure 3. The left-hand figure shows the real part of the poles and zeros of the 2-DOF model given in (21) for straight-running vehicle speeds between $20 \mathrm{~m} / \mathrm{s}$ and $80 \mathrm{~m} / \mathrm{s}$. The poles are the roots of the characteristic polynomial given in (22). The zeros of the lateral acceleration, side-slip angle and yaw velocity transfer functions are computed using (24), (25) and (26), respectively. In the case of the lateral acceleration, the zeros form a complex conjugate pair. The right-hand figure shows the responses of the lateral acceleration, side-slip angle and yaw velocity transfer functions to step changes in the steering input. These responses are computed at longitudinal speeds of $u_{0}=\{30 \mathrm{~m} / \mathrm{s}, 50 \mathrm{~m} / \mathrm{s}, 70 \mathrm{~m} / \mathrm{s}\}$ corresponding respectively to the magenta, green and cyan curves.

\subsection{Accelerating and Braking Stability}

It is shown in [13] that the vehicle model given here can be considered slowly time varying and therefore that the eigenvalues can be used to assess the vehicle's stability. As the vehicle accelerates (or decelerates), the frozen-time eigenvalues are computed along the manoeuvre and used to assess the time-localised stability of the car. These findings are then correlated with the time response in the yaw velocity.

\subsubsection{Closed-Form Solution}

The first-order differential equations (12) and (13) can combined by eliminating the lateral velocity to produce a second-order differential equation in the yaw rate $\omega$. The analysis is further simplified by assuming that the steering angle is held at zero in order to obtain

$$
u(t)^{2} \ddot{\omega}+P u(t) \dot{\omega}+\left(Q u(t)^{2}+R\right) \omega=0,
$$

where the parameters $P, Q$ and $R$ are given by

$$
P=\frac{-k^{2} m a_{x}+\left(a^{2}+k^{2}\right) C_{f}+\left(b^{2}+k^{2}\right) C_{r}}{m k^{2}}, \quad Q=-\frac{C s}{m k^{2}}, \quad R=\frac{l^{2} C_{f} C_{r}}{m^{2} k^{2}} .
$$

This time-varying differential equation has a structure reminiscent of a Bessel's differential equation and so a closed-form solution can be found in terms of Bessel functions. As demonstrated in [14] an equation with the form:

$$
u^{2} \frac{d^{2} \omega}{d u^{2}}+P u \frac{d \omega}{d u}+\left(Q u^{2}+R\right) \omega=0
$$

has a solution

$$
\omega=c_{1} u^{\rho}(t) J_{\nu}(\alpha u(t))+c_{2} u^{\rho}(t) Y_{\nu}(\alpha u(t)),
$$


in which $J_{v}$ and $Y_{v}$ are Bessel functions of the first and second kind, and $c_{1}$ and $c_{2}$ are constants of integration. Comparing equations (27) and (29) show that a change of variable from $t$ to $\omega$ is needed. The first- and second-order derivatives of $\omega$ with respect to $u$ are

$$
\frac{d \omega}{d t}=\frac{d \omega}{d u} \frac{d u}{d t}=a_{x} \frac{d \omega}{d u}
$$

and

$$
\frac{d^{2} \omega}{d t^{2}}=\frac{d^{2} \omega}{d u^{2}}\left(\frac{d \omega}{d u}\right)^{2}+\frac{d \omega}{d t} \frac{d^{2} u}{d t^{2}}=a_{x}^{2} \frac{d^{2} \omega}{d u^{2}} .
$$

The parameters $\rho$ and $\alpha$, and the order of the Bessel functions $\nu$, can now be related to the coefficients of the differential equation and $a_{x}$ by:

$$
\rho=\frac{1-\frac{P}{a_{x}}}{2}, \quad \alpha=\sqrt{\frac{Q}{a_{x}^{2}}}, \quad \nu=\sqrt{\frac{\left(1-\frac{P}{a_{x}}\right)^{2}}{4}-\frac{R}{a_{x}^{2}}} .
$$

If $Q$ is negative the argument of the Bessel functions is complex. In this case, in order to obtain real solutions, modified Bessel functions $I_{v}$ and $K_{v}$ of the first and second kind must be used to obtain

$$
\omega=c_{1} u^{\rho}(t) I_{\nu}(\alpha u(t))+c_{2} u^{\rho}(t) K_{\nu}(\alpha u(t)) .
$$

The sign of $Q$ depends on the sign of $C s$. However, unlike the constant velocity case, this quantity now changes with both the car kinematic parameters and the vehicle's acceleration. For example, a car might be oversteering at a constant velocity $(C s<$ 0 ), however when the vehicle accelerates, normal load is transferred to the rear and $C_{r}$ increases, while $C_{f}$ simultaneously decreases thereby producing possibly a negative value of $C s$. Under braking the opposite occurs and $C s$ will remain negative (or may become negative if the vehicle is initially under-steering). As the sign of $C s$ changes the appropriate Bessel functions must be used.

The constants of integration, $c_{1}$ and $c_{2}$ are found by applying the initial conditions $\omega(0)=\omega_{0}$ and $\dot{\omega}(0)=\dot{\omega}_{0}$. Clearly

$$
\omega_{0}=u_{0}^{\rho}\left(c_{1} J_{\nu}\left(\alpha u_{0}\right)+c_{2} Y_{\nu}\left(\alpha u_{0}\right)\right) .
$$

The derivative of the general solution is required to apply the second initial condition:

$$
\begin{aligned}
& a_{x} \dot{\omega}_{0}=c_{1}\left[\rho u_{0}^{\rho-1} J_{\nu}(\right.\left.\left.\alpha u_{0}\right)+u_{0}^{\rho}\left(J_{\nu-1}\left(\alpha u_{0}\right)-\frac{\nu}{\alpha u_{0}} J_{\nu}\left(\alpha u_{0}\right)\right) \alpha\right] \\
&+c_{2}\left[\rho u_{0}^{\rho-1} Y_{\nu}\left(\alpha u_{0}\right)+u_{0}^{\rho}\left(Y_{\nu-1}\left(\alpha u_{0}\right)-\frac{\nu}{\alpha u_{0}} Y_{\nu}\left(\alpha u_{0}\right)\right) \alpha\right] .
\end{aligned}
$$

If the velocity is assumed constant the yaw differential equation reduces to a linear second-order time-invariant equation with solution

$$
\omega=c_{1} e^{\lambda_{1} t}+c_{2} e^{\lambda_{2} t},
$$



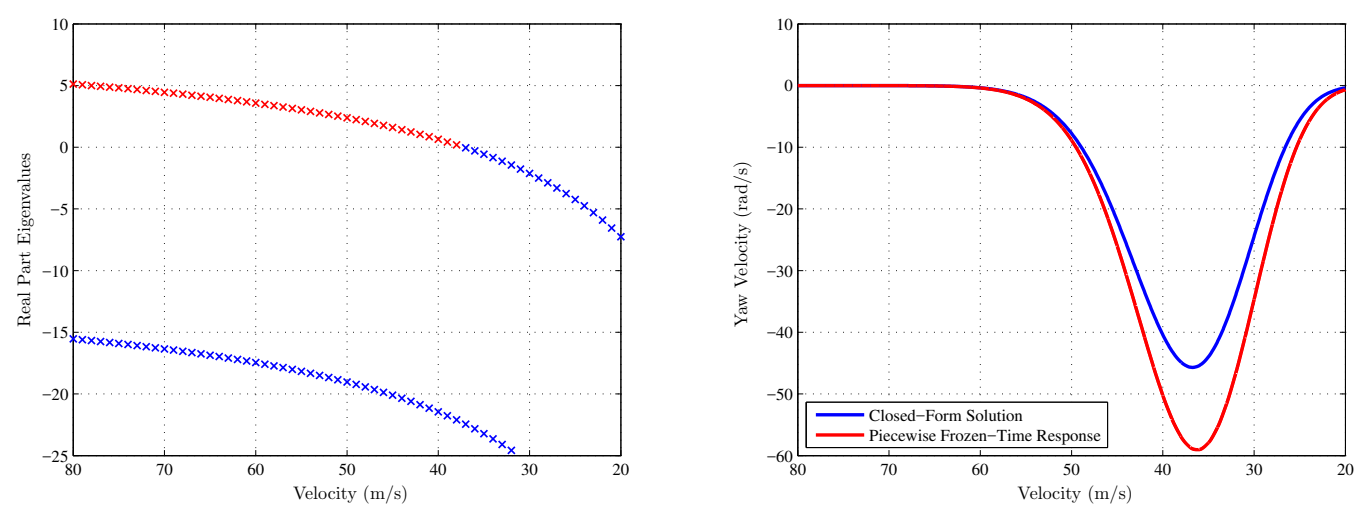

Figure 4. The left-hand figure shows the real parts of the frozen-time eigenvalues of a vehicle braking in straight running at $-10 \mathrm{~m} / \mathrm{s}^{2}$ for vehicle speeds from $80 \mathrm{~m} / \mathrm{s}$ to $20 \mathrm{~m} / \mathrm{s}$. The right-hand figure shows the yaw response to a non-zero initial yaw velocity at $80 \mathrm{~m} / \mathrm{s}$.
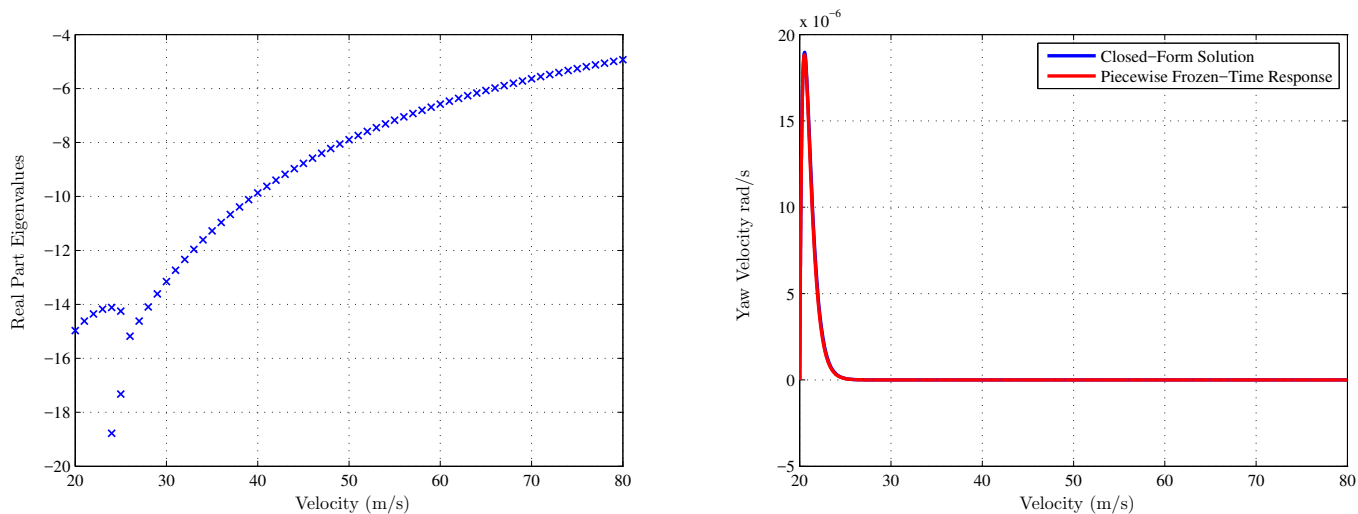

Figure 5. The left-hand figure shows the real part of the frozen-time eigenvalues of a vehicle accelerating in straight running at $10 \mathrm{~m} / \mathrm{s}^{2}$ from $20 \mathrm{~m} / \mathrm{s}$ to $80 \mathrm{~m} / \mathrm{s}$. The right-hand figure shows the yaw response to a non-zero initial yaw velocity at $20 \mathrm{~m} / \mathrm{s}$.

where $\lambda_{1}$ and $\lambda_{2}$ are the model eigenvalues. The constants $c_{1}$ and $c_{2}$ can be found, once again, through the application of the initial conditions. The time-invariant yaw response will differ from the time-varying solution over long time periods, and a piecewise time-invariant yaw response can therefore be used. Suppose that time is divided into intervals, and that the time-invariant solution is computed on each interval with the terminal conditions from the previous interval used as the initial conditions for the current interval. As these sub-intervals decrease in duration the solution accuracy, as expected, improves.

In the case where $C s$ is negative, the eigenvalues are complex, and a real solution is found from the general solution of the form:

$$
\omega=c_{1} e^{\lambda_{r} t} \cos \left(\lambda_{i} t\right)+c_{2} e^{\lambda_{r} t} \sin \left(\lambda_{i} t\right),
$$

where, $\lambda_{r}$ and $\lambda_{i}$ are the real and imaginary parts of the eigenvalues.

\subsubsection{Results}

The eigenvalues and system response for a constant $-10 \mathrm{~m} / \mathrm{s}^{2}$ of braking are shown in Figure 4 , for speeds varying from $80 \mathrm{~m} / \mathrm{s}$ to $20 \mathrm{~m} / \mathrm{s}$. One of the eigenvalues changes sign 
at $V_{\text {crit }}=38 \mathrm{~m} / \mathrm{s}$, which is much lower than the constant velocity case $(65 \mathrm{~m} / \mathrm{s})$. The load transfer due to braking reduces the rear tire cornering stiffness. This, in turn, causes $C s$ to increase with an accompanying reduction in the critical speed; see (23). The response of the time-varying system to a non-zero initial yaw velocity at $80 \mathrm{~m} / \mathrm{s}$ is determined using the closed-form solution in (30). Initially, the yaw angle grows exponentially, typical of an unstable system. However, as the vehicle speed decreases, and the eigenvalues become negative, the yaw angle response slows and then decays. This behaviour suggests that a vehicle braking from $80 \mathrm{~m} / \mathrm{s}$ may exhibit an undesirable 'burst' response. Under acceleration the eigenvalues of the system remain negative, and indeed, the system response to a non-zero initial condition exhibits a stable characteristic. The piecewise frozen-time solution predicts almost exactly the real solution. At higher speeds the eigenvalues are complex, which manifests in an underdamped yaw response to a perturbation.

\section{Cornering Stability}

The straight-running analysis is now extended to the case of a cornering vehicle for both the constant and variable speed cases.

\subsection{Constant Velocity}

Under cornering conditions the longitudinal and lateral dynamics given in (1) and (2) interact. Furthermore, in steady-state cornering, the tire side-slip angles are no longer zero and the linear tire model given in (10) is no longer usable. Instead, combined-slip magic formula type tires must be used. Empirical formulae, given in [15] determine the magnitude of the lateral and longitudinal tire forces as functions of the tire side-slip angle, longitudinal slip and normal load, and can be represented as:

$$
F_{x}=g\left(\alpha, \kappa, F_{z}\right), \quad F_{y}=f\left(\alpha, \kappa, F_{z}\right) .
$$

If the EOM in (1), (2), and (3) are linearised about the steady-state (constant velocity and radius-of-term) operating point, the state-space model is given by:

$$
\begin{gathered}
A=\left(\begin{array}{ccc}
\frac{-C_{f} \delta_{0}\left(v_{0}+a \omega_{0}\right)}{m u_{0}^{2}} & \frac{C_{f} \delta_{0}+m u_{0} \omega_{0}}{m u_{0}} & \frac{C_{f} a \delta_{0}+m u_{0} v_{0}}{m u_{0}} \\
\frac{C v_{0}+\left(C s-m u_{0}^{2}\right) \omega_{0}}{m u_{0}^{2}} & -\frac{C}{m u_{0}} & \frac{-C s-m u_{0}^{2}}{m u_{0}} \\
\frac{C s v_{0}+C q^{2} \omega_{0}}{J u_{0}^{2}} & \frac{-C s}{J u_{0}} & -\frac{C q^{2}}{J u_{0}}
\end{array}\right) B=\left(\begin{array}{c}
-\frac{C_{f} \delta_{0}+F_{f y 0}}{m} \\
\frac{C_{f}-F_{f y 0} \delta_{0}}{m} \\
\frac{C_{f} a-F_{f y 0} a \delta_{0}}{J}
\end{array}\right) \\
C=\left(\begin{array}{ccc}
\frac{C s \omega_{0}+v_{0} C}{m u_{0}^{2}} & -\frac{C}{m u_{0}} & -\frac{C s}{m u_{0}} \\
0 & 0 & 1 \\
-\frac{v_{0}}{u_{0}^{2}} & \frac{1}{u_{0}} & 0
\end{array}\right) \quad D=\left(\begin{array}{c}
\frac{C_{f}-F_{f y 0} \delta_{0}}{m} \\
0 \\
0
\end{array}\right) .
\end{gathered}
$$

The outputs are again $\left[a_{y}, \beta, \omega\right]$, where the simplifications in (16) to (19) are used and the linearisation of the front tire forces is given by:

$$
\left[\begin{array}{l}
\frac{\partial F_{f y}}{\partial u} \\
\frac{\partial F_{f y}}{\partial v} \\
\frac{\partial F_{f y}}{\partial \omega_{f y}} \\
\frac{\partial F_{f y}}{\partial \delta}
\end{array}\right]=\left.\frac{\partial F_{f y}}{\partial \alpha_{f}}\right|_{\alpha_{f 0}, \kappa_{f 0}, F_{f z 0}}\left[\begin{array}{c}
\frac{\partial \alpha_{f}}{\partial u} \\
\frac{\partial \alpha_{f}}{\partial v} \\
\frac{\partial \alpha_{f}}{\partial \omega_{f}} \\
\frac{\partial \alpha_{f}}{\partial \delta}
\end{array}\right]_{u_{0}, v_{0}, \omega_{0}, \delta_{0}}=C_{f}\left[\begin{array}{c}
\frac{\omega_{0} a+v}{u^{2}} \\
-\frac{1}{u_{0}} \\
-\frac{a}{u_{0}} \\
1
\end{array}\right]
$$



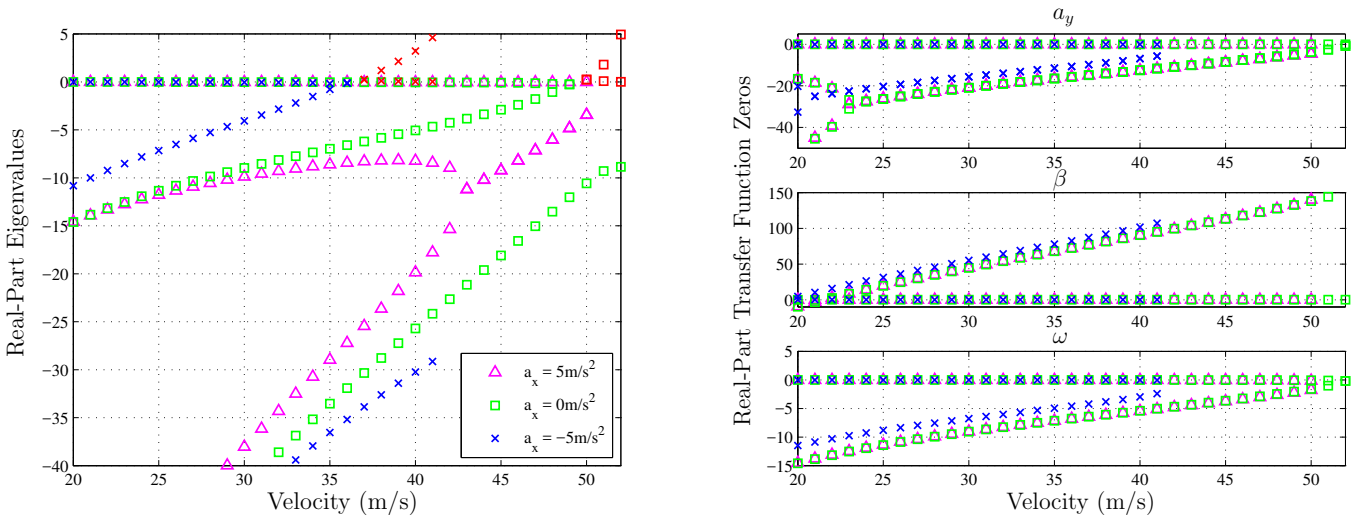

Figure 6. The left-hand figure shows the real-part of the eigenvalues of the 3-DOF vehicle model plotted for different vehicle speeds at vehicle accelerations of $\mathrm{a}_{\mathrm{x}}=5 \mathrm{~m} / \mathrm{s}^{2}, 0 \mathrm{~m} / \mathrm{s}^{2},-5 \mathrm{~m} / \mathrm{s}^{2}$, . The right-hand figure shows the zeros of each of the transfer functions across different speeds and at the different acceleration levels.

The rear-tire force is treated similarly.

The operating conditions required to evaluate the linearised model are determined for a steady-state velocity $(V)$ and radius-of-turn $(R)$. Once $V$ and $R$ have been chosen, $\omega$ is determined by the kinematic relation:

$$
\omega=\frac{V}{R} .
$$

The resultant vehicle velocity is related to the lateral and longitudinal velocities by:

$$
V^{2}=v^{2}+u^{2} .
$$

Under steady-state conditions, the EOM reduce to the following algebraic relations:

$$
\begin{aligned}
& 0=m \omega_{0} v_{0}-F_{f y 0} \delta_{0}+F_{r x 0} \\
& 0=-m \omega_{0} u_{0}+F_{f y 0} \cos \delta_{0}+F_{r y 0} \\
& 0=a F_{f y 0} \cos \delta_{0}-b F_{r y 0} .
\end{aligned}
$$

These five equations and the non-linear tire model are solved simultaneously to determine the steady-state values of $u_{0}, v_{0}, \omega_{0}, \delta_{0}, F_{f y 0}, F_{r y 0}$, as well as $F_{r x 0}$, which is a small driving force required to maintain the vehicle at a constant velocity. The solution is used to evaluate the stability of the equilibrium point and to determine the response of the outputs to perturbations in the steering input.

The parameters of the vehicle studied are given in Table A1 and the normalised tire characteristics are given in the Appendix. The vehicle stability is determined for speeds ranging from $\mathrm{V}=20 \mathrm{~m} / \mathrm{s}$ to $\mathrm{V}=53 \mathrm{~m} / \mathrm{s}$ in a constant $170 \mathrm{~m}$ radius turn. At speeds above $\mathrm{V}=53 \mathrm{~m} / \mathrm{s}$ the tires saturate and the vehicle can no longer maintain the corner. The real-parts of the system eigenvalues are plotted at different vehicle speeds in the left half of Figure 6. As the speed increases the eigenvalues migrate towards, and ultimately into the right-half plane. From the 2-DOF analysis, this indicates that the vehicle is over steering, which is consistent with the centre-of-mass being located towards the rear of the vehicle. In contrast to the 2-DOF model, in this case both the location and type of eigenvalue change. Initially, there are three negative real eigenvalues, which migrate towards the imaginary axis as the speed increases. Two of these eigenvalues then form 

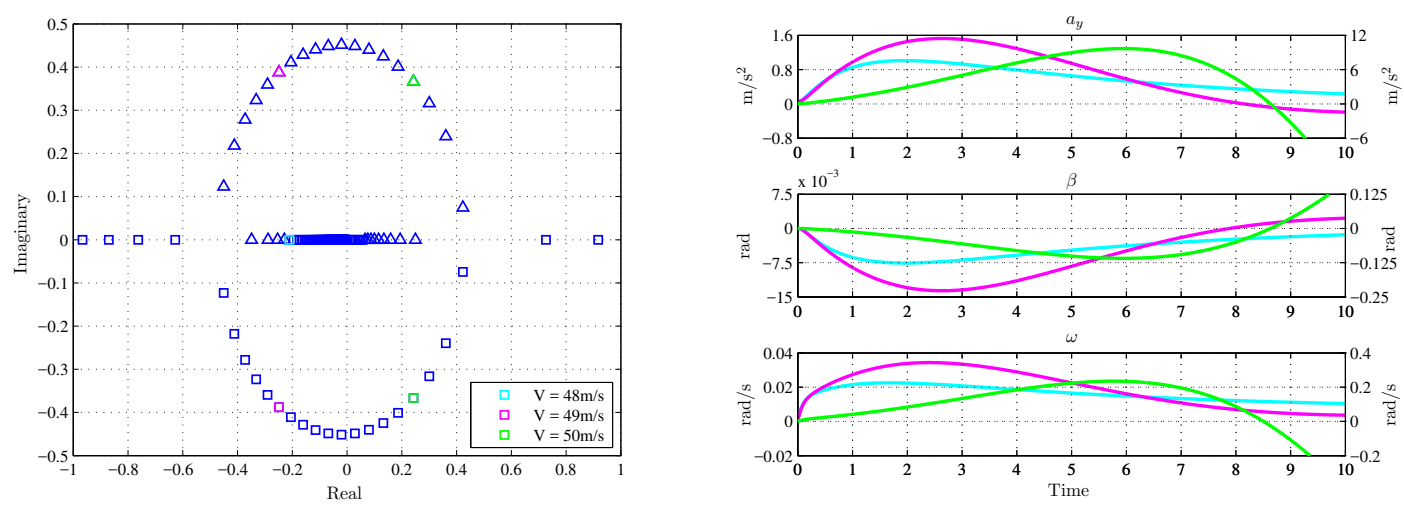

Figure 7. The left-hand figure shows the root-locus plot for two of the three eigenvalues of the 3-DOF vehicle model under cornering; the varied parameter is the vehicle's speed. The eigenvaalues corresponding to the longitudinal speeds $\mathrm{V}=\{48 \mathrm{~m} / \mathrm{s}, 49 \mathrm{~m} / \mathrm{s}, 50 \mathrm{~m} / \mathrm{s}\}$ are highlighted in cyan, magenta and green, respectively. The right-hand figure shows the step responses of the 3-DOF model at the listed longitudinal speeds. The top plot shows the lateral acceleration responses to a step in steering angle, the centre plot shows the body side-slip angle response, while the bottom plot illustrates the yaw rate response. The cyan and magenta curves are associated with the scale on the left axes, while the green curves with those on the right.

a complex conjugate pair that moves across the imaginary axis. This is shown in more detail in the root-locus plot of Figure 7. The right half of Figure 6 shows the zeros of each of the three transfer functions. Each has a zero at the origin, which implies zero steadystate gain. The side-slip angle response contains a non-minimum phase zero, similar to the 2-DOF model, this is correlated with the system responses in Figure 7.

In Figure 7 the system response is computed at $\mathrm{V}=\{48 \mathrm{~m} / \mathrm{s}, 49 \mathrm{~m} / \mathrm{s}, 50 \mathrm{~m} / \mathrm{s}\}$; the eigenvalue type is different at each speed. At $\mathrm{V}=48 \mathrm{~m} / \mathrm{s}$ all three responses exhibit overdamped behaviour, consistent with real, negative eigenvalues. When the eigenvalues become complex at $\mathrm{V}=49 \mathrm{~m} / \mathrm{s}$ and $\mathrm{V}=50 \mathrm{~m} / \mathrm{s}$ the corresponding responses become underdamped and oscillatory. When the system is still stable at $\mathrm{V}=49 \mathrm{~m} / \mathrm{s}$ the oscillations decay, however, once the system is unstable at $\mathrm{V}=50 \mathrm{~m} / \mathrm{s}$ the responses grow. Once again, the side-slip response is differentiated from the yaw velocity by the non-minimum phase behaviour resulting from the right-half plane zero.

\subsection{Accelerating and Braking Under Cornering}

Straight-line accelerating and braking, and steady-state cornering only represent the axes of the G-G diagram. To determine the stability over the rest of the operating regime it is necessary to develop a method of examining acceleration and braking under cornering. In this scenario, the vehicle is no longer in equilibrium, since the states are changing with time. It is possible to study the stability of an accelerating or braking vehicle by examining perturbations from some nominal motion [16]. To conduct this type of analysis the vehicle states along a nominal prescribed trajectory are required. These evolving states can be found by simulating the manoeuvre using the non-linear vehicle model with feedback controllers that are used to generate the plant inputs that produce the required motion. This approach has a number of limitations: simulating the manoeuvre is a time-consuming process and determining vehicle stability is intended to be a way predicting the vehicle's response without having to numerically integrate the equations of motion! Furthermore, as the vehicle becomes unstable, and the braking and acceleration becomes more aggressive, the feedback controllers are less able to maintain the vehicle on the reference trajectory. 

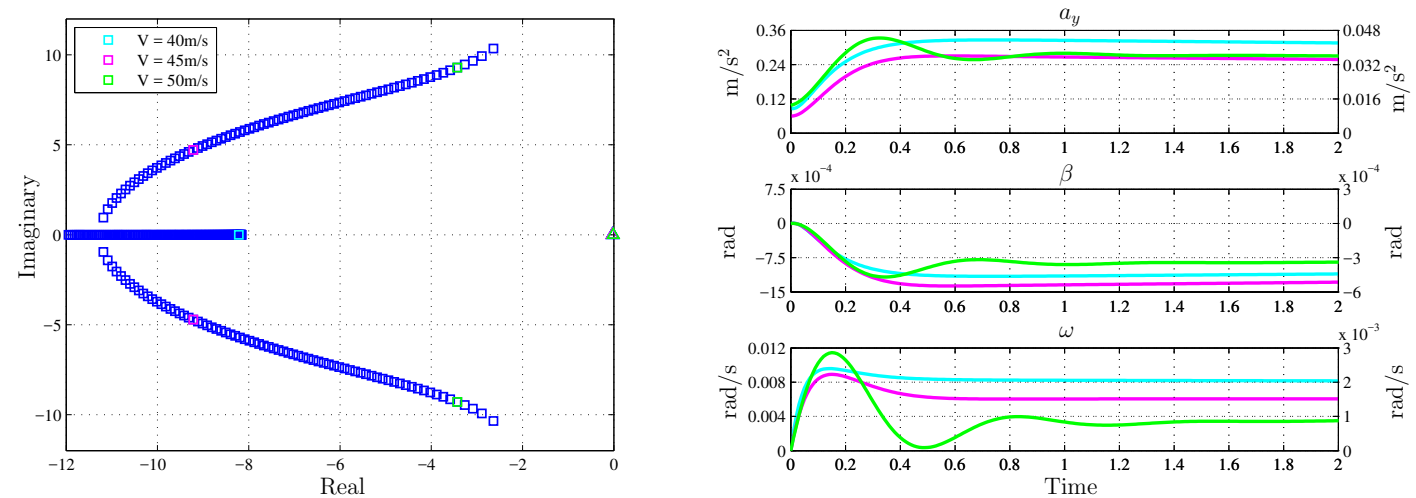

Figure 8. The left-hand figure shows the root-locus plot of the eigenvalues of the 3-DOF vehicle model under $5 \mathrm{~m} / \mathrm{s}^{2}$ acceleration. At low speeds there are three negative real eigenvalues. As the speed increases, the pole near the origin remains there, whilst the other two move together, coalesce, and form a complex-conjugate pair. While the complex conjugate pair move towards the imaginary axis as the vehicle speed increases, the car loses traction before they cross into the right-half plane. The eigenvalues corresponding to speeds of $\mathrm{V}=\{40 \mathrm{~m} / \mathrm{s}, 45 \mathrm{~m} / \mathrm{s}, 50 \mathrm{~m} / \mathrm{s}\}$ are highlighted in cyan, magenta and green, respectively. The right-hand figure shows the step response plots for the 3-DOF model at the cited longitudinal speeds. From top to bottom, the plots show the lateral acceleration, the side-slip angle and the yaw velocity responses to a step change in the steering angle. The green curves are associated with the right-hand axes, while the cyan and magenta curves are associated with the left-hand axes.

An alternative approach to determining the frozen-time vehicle states is to use a D'Alembert force system that acts as a surrogate for the real forces of inertia experienced under acceleration. In this approach, an accelerating or braking vehicle is approximated by one travelling at a constant velocity, but subjected to forces that capture the inertial effects of accelerating and braking. The advantage of this method is clear. The vehicle remains in equilibrium and the vehicle states can be determined from algebraic equations of motion, which now contain additional terms representing the apparent forces. The frozen-time eigenvalues of the system are computed at each point along a motion trajectory. The choice of manoeuvres used to 'fill out' the G-G diagram is not unique.

For example, the vehicle could be braked while the radius of turn decreases, to maintain a constant lateral acceleration. This would correspond to a line on the G-G diagram that is parallel to the longitudinal acceleration axis. A more common physical scenario, and the one studied here, is the vehicle braking or accelerating in a constant $(170 \mathrm{~m})$ radiusof-turn bend.

Once again, the limitation of this eigenvalue-based approach is that stability can only be inferred from the frozen-time system eigenvalues if the system is slowly time-varying.

In Figure 6 the eigenvalues and zeros of the vehicle model are computed for acceleration and braking of $\pm 5 \mathrm{~m} / \mathrm{s}^{2}$. In both cases the achievable top speed of the vehicle is reduced. When the vehicle is driven or braked, the torque applied at the rear wheels increases the tire longitudinal slip angle, which reduces the ability of the tire to generate lateral force. Under accelerating there is a normal load transfer to the rear tires, this changes the vehicle from oversteering to understeering. Consequently, vehicle remains stable to the point where the tires lose traction. Under braking the load transfer to the front axle reduces the critical speed and the vehicle becomes unstable at $37 \mathrm{~m} / \mathrm{s}$.

The responses of the vehicle outputs to a step steering angle input is once again examined at different vehicle speeds. In Figure 8 the eigenvalue root-locus diagram for the accelerating vehicle is given and the step-responses at the highlighted eigenvalues computed; these correspond to speeds of $\mathrm{V}=\{40 \mathrm{~m} / \mathrm{s}, 45 \mathrm{~m} / \mathrm{s}, 50 \mathrm{~m} / \mathrm{s}\}$. The acceleration responses differ significantly from the constant velocity case given in Figure 7. Firstly, the responses reach steady-state more quickly in the accelerating case. Secondly, the re- 

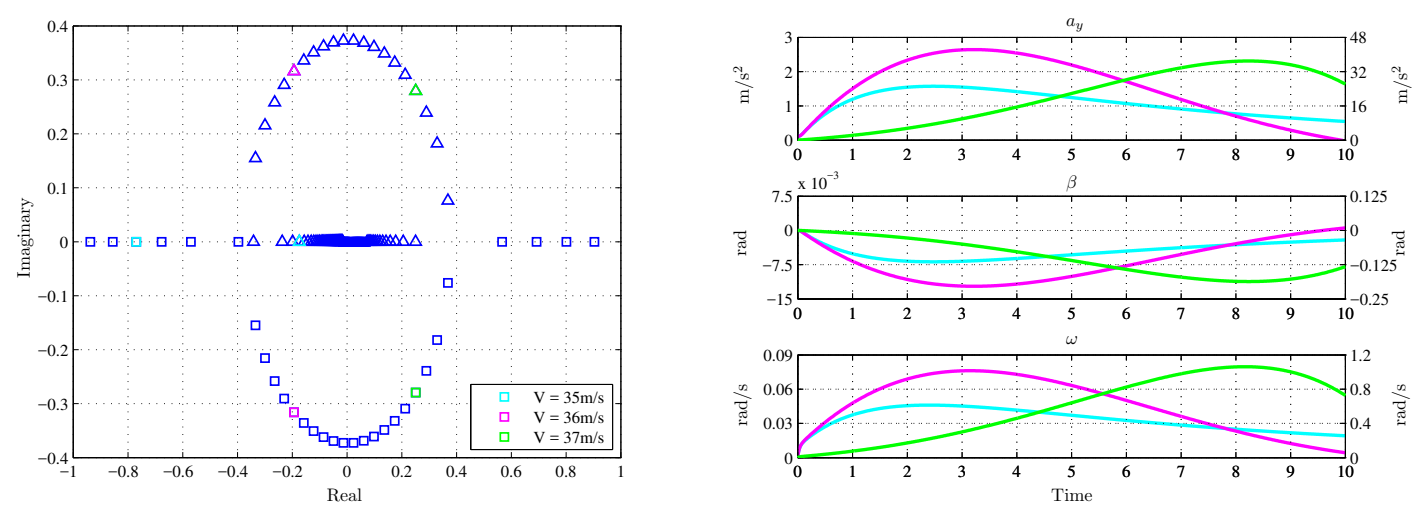

Figure 9. The left figure contains the root-locus plot of two of the eigenvalues of the 3-DOF vehicle model under $-5 \mathrm{~m} / \mathrm{s}^{2}$ braking. As the vehicle speed increases, one pole moves away from the origin as the other moves towards it. These then coalesce and form a complex conjugate pair that migrates into the right-half plane. At higher speeds these become two positive real poles, with one moving towards the origin and the other away from it. The eigenvalues corresponding to the longitudinal speeds $\mathrm{V}=\{35 \mathrm{~m} / \mathrm{s}, 36 \mathrm{~m} / \mathrm{s}, 37 \mathrm{~m} / \mathrm{s}\}$ are highlighted in cyan, magenta and green, respectively. The right figure contains the step response plots for a 3-DOF model, computed at the listed longitudinal speeds. From top to bottom, the sub-plots contain the responses of the lateral acceleration, body side-slip angle and yaw velocity transfer functions to a step in steering angle. The cyan and magenta curves are associated with the scale on the left axes, while the green curves with those on the right.

sponses are more oscillatory in the accelerating case, with the damping factor decreasing as the speed increases. Thirdly, the steady-state responses are much smaller in the accelerating case, with the step-response magnitudes becoming smaller at higher speeds. Thus under acceleration at high speeds, the responsiveness of the vehicle to steering inputs reduces. In [5] it is noted that as an understeering vehicle approaches the limit of adhesion control moments which change the orientation of the vehicle can no longer be generated with front steer. The vehicle's braking responses and stability characteristics shown in Figure 9 are in several ways similar to the steady-state behaviours given in Figure 7 . The root loci and the vehicle responses bear a strong resemblance to the constant velocity case, although the limit responses occur at lower vehicle speeds. While the braking and constant-speed responses have similar general shapes, the vehicle is more responsive in lateral acceleration and yaw than it is in the constant velocity case.

This study illustrates clearly the changes in vehicle behaviour under accelerating and braking (as opposed to constant speed), and highlights the need for stability and vehicle responsiveness to be analysed across the full G-G diagram when contemplating the design of simulator cueing filters.

\section{Near-Limit Cueing}

The above analysis shows that the lateral and yaw responses of the vehicle, and its over and understeer characteristics, vary significantly with the operating condition. If the simulator driver is to operate the vehicle on its performance limit under cornering, and assess continuously the vehicle's handling behaviour, these changes must be cued. In cornering, the lateral and yaw motion cues must be examined simultaneously, since in concert, they create the sensation of rotating about a centre of turning. The location of the centre of turning varies continuously and is strongly influenced by the car's over and understeer characteristics; these changes should be considered in the design and tuning of cueing algorithms. The results of the stability analysis are discussed in the cueing context in Section 4.1. Changes in the centre of turning under cornering are discussed in 
Section 4.2 .

\subsection{Vehicle Limit Behaviour}

Sections 2 and 3 examine vehicle stability and response changes under different operating conditions. This information can be used to determine the proximity of the car to the performance limit, and these behavioural changes must be cued to the simulator driver by appropriate platform motions.

In constant-speed straight-running the stability of the vehicle depends on the car set-up and the speed. As shown in Section 2.2, the set-up produces either an under or oversteering vehicle, with the oversteering car becoming unstable above the critical speed. In the simulator the driver needs to identify the car's steering characteristics, as well as its proximity to the stability limit. As shown in Figure 3, the vehicle speed of response to steering inputs increases as the vehicle becomes progressively less stable. To assist the driver, the simulator motion cues should recreate these larger magnitude motions, reduced response times and reduced damping ratios as the car approaches the stability limit.

When the vehicle is subjected to changes in the longitudinal acceleration or braking, there is a corresponding normal load transfer between its front and rear axles. These normal load variations affect the tire characteristics and thus the steering behaviour of the vehicle. As shown in Section 2.3, the accelerating car becomes understeering and therefore stable at all speeds. The critical speed is reduced in the braking case. Under acceleration, a perturbation in the yaw acceleration produces a short burst response in the yaw velocity which decays rapidly. However, under braking, the yaw response to a perturbation will increase until the vehicle is slowed below the critical speed. Cues that communicate these behavioural changes must be present in the simulator so that the driver can keep track of the effects of acceleration and braking.

In steady-state cornering the vehicle's response to steering inputs changes significantly with speed and acceleration/braking. At constant low speeds there are three stable real eigenvalues. As the speed increases, and the car approaches the stability limit, the vehicles response becomes oscillatory with reduced damping. This behaviour should also be cued by the motion platform, since it indicates that the vehicle is approaching the critical speed.

When the car accelerates it begins to understeer and exhibit an oscillatory response. In this situation the driver must find the tire limit so that the corner can be maintained at high speed. As the vehicle approaches the tire adhesion limit, the gains from steering input to the vehicle's outputs (lateral acceleration, yaw velocity and side-slip) decrease as does the damping. These changes must be also communicated to the simulator driver so that he/she is forewarned of their approach to the handling limit of the car.

The cues required by the driver when braking in a turn are similar to those required at constant speed, although the car is more responsive to steering inputs. This increase in gain must be detectable in the simulator. The higher damping factors and the reduced overshoot in the responses should also be cued.

The side-slip angle behaviour is key in cueing vehicle stability. Following a disturbance input to a stable car the side-slip angle returns to zero thereby re-aligning the vehicle velocity with its longitudinal direction [5]. The side-slip angle is also an indicator of the proximity of the tires to lateral force saturation. This side-slip angle derived information is useful to the driver in his/her efforts to push the vehicle to its performance limit. 

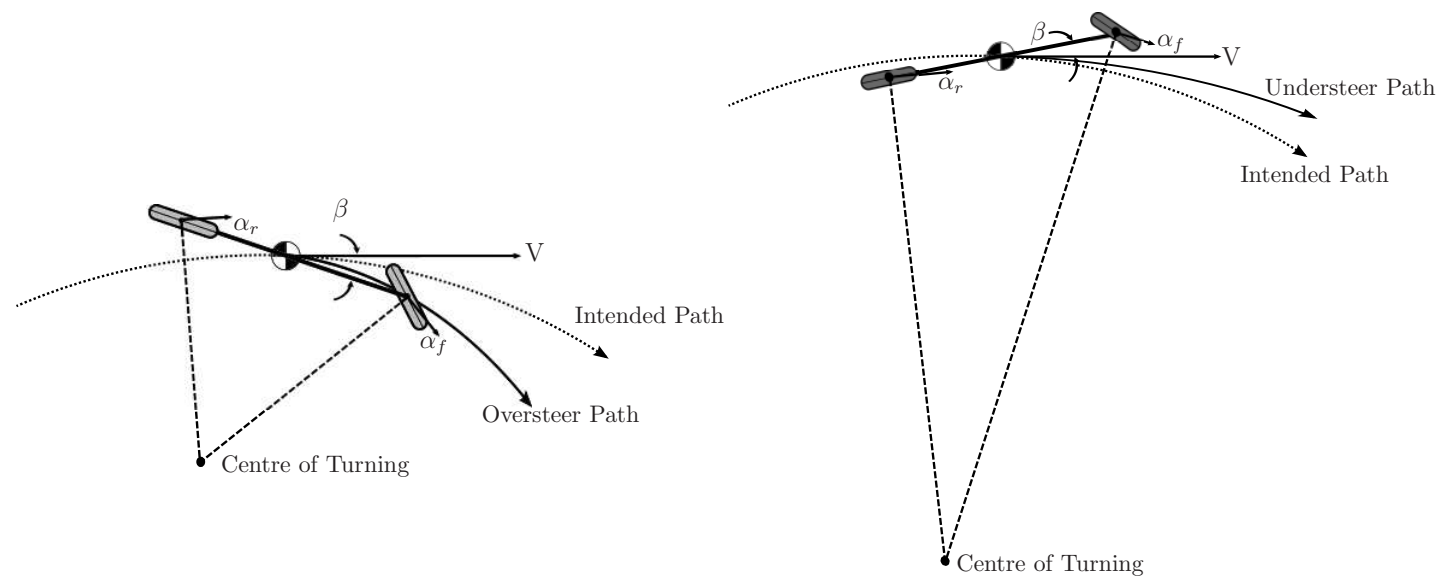

Figure 10. The kinematics of an oversteering vehicle navigating a turn are illustrated in the left-hand figure, while those of an understeering vehicle are illustrated in the right-hand diagram.

\subsection{Centre of Turning}

The centre of turning is a point in the reference frame of the vehicle that is absolutely stationary. One way to find the centre of turning is to determine the intersection point of perpendiculars to the front- and rear-tire ground contact point velocity vectors. This point moves as the front- and rear-tire slip angles change. The centre of turning can also be found in terms of the vehicle's longitudinal, lateral and yaw velocities.

The absolute velocity of a general point, $\boldsymbol{P}$, in the vehicle's reference frame is given by

$$
\boldsymbol{v}_{P}=\boldsymbol{P} \times \boldsymbol{\omega}+\boldsymbol{v}_{c a r},
$$

where $\boldsymbol{v}_{c a r}$ is the car's absolute velocity. The centre of turning is a point $\boldsymbol{P}_{t}$, which is absolutely stationary, and therefore given by

$$
0=\boldsymbol{P}_{t} \times \boldsymbol{\omega}+\boldsymbol{v}_{c a r},
$$

or

$$
\left(\begin{array}{c}
P_{t x} \\
P_{t y}
\end{array}\right)=\frac{1}{\omega}\left(\begin{array}{c}
v \\
-u
\end{array}\right) .
$$

The components of $\boldsymbol{P}_{t}$ are $P_{t x}$ and $P_{t y}$, which are the distances of the rotation centre from origin of the car-fixed coordinate system. It is clear that

$$
P_{t x}^{2}+P_{t y}^{2}=\frac{u^{2}+v^{2}}{\omega^{2}}=\frac{V^{2}}{\omega^{2}}=R^{2},
$$

where $R$ is the radius of turn.

The locations of the centres of turning for oversteering and understeering vehicles are compared in Figure 10. When navigating a turn, a vehicle is said to exhibit oversteer if $R_{\text {int }}>R$, where $R_{\text {int }}$ is the intended radius of turn. The vehicle tends to steer inside the intended path (oversteer) if there is less grip available at the rear of the car than at the front.

Understeering is associated with reduced front-tire grip. In this case the vehicle will tend to run outside the intended path, with the front slip angle increasing more rapidly 

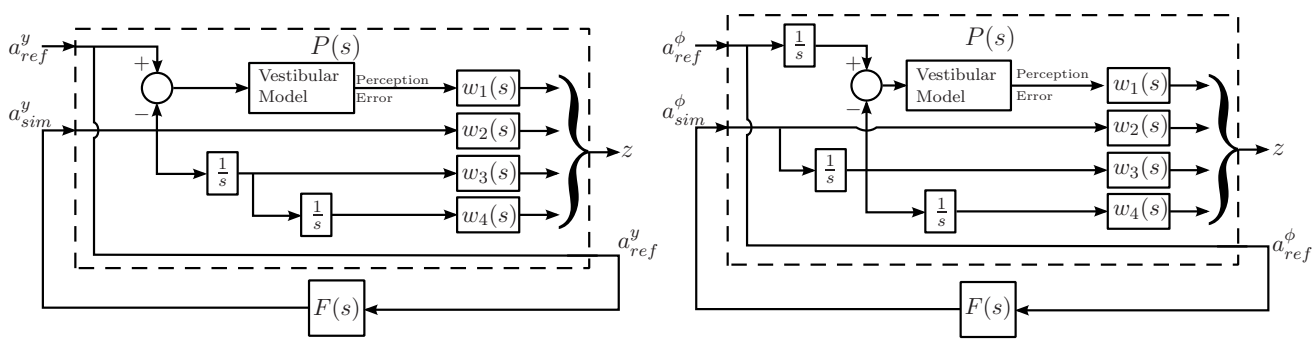

Figure 11. Lateral acceleration and yaw cueing filter design problems cast in the generalised regulator framework $[3,17]$.

than that at the rear.

Changes in the perceived location of the centre of turning is important in the simulator. Although it is not possible to create a sustained sensation of cornering, miscues that create a false perception of the centre of turning location can lead to misinterpretations of the vehicle's behaviour.

\section{Cueing Filter Design}

The optimal-control-based cueing filter design strategy is frequently used in passenger car applications [4], and is applied to the longitudinal race-car motion in [3]. In this work, this method is used to design filters for the lateral and yaw directions, which are tuned using the analysis of the vehicle limit behaviour presented in the previous sections.

\subsection{Lateral Filter Design}

A design framework for an optimal lateral cueing filter is given in Figure 11. The problem is cast in the generalised regulator framework [17], where the plant output is given by $z$, the input is $a_{r e f}^{y}$, the input to the filter is $a_{r e f}^{y}$ and the filter output is $a_{s i m}^{y}$. The cueing filter $F(s)$ is designed to minimise the $\mathcal{H}_{2}$-norm from $a_{\text {ref }}^{y}$ to $z$.

An inherent limitation of any simulator is its inability to reproduce sustained accelerations, and so only the high-frequency changes in the motion are cued. Typically, the simulator will produce some onset motion at the beginning of a manoeuvre, which will quickly decay, and thereafter the visual aids become the dominant source of information. The platform will then be moved back to the centre of the workspace in preparation for the following motion that could be in any direction. This 'washout' effect is a commonly used strategy to prevent the platform reaching the workspace limits.

In the optimal filter design framework the washout effect is produced by including the appropriate platform acceleration, velocity and displacement in the output $z$. The other element of $z$ is the perceived acceleration error. The minimisation of the platform motion and the acceleration error are conflicting objectives, and weighting functions are used to produce frequency-dependent trade-offs and thus different platform cueing strategies.

The design of the yawing filter follows a similar process to that used in the design of the lateral acceleration filter, with various different approaches presented in the literature [4]. Within the capabilities of the motion platform, the filter is again designed to minimise the yaw velocity error, since humans can sense the vehicle's angular velocity.

It is important to ensure that coherent yaw velocity and lateral acceleration cues are supplied to the motion platform. The yaw acceleration filter is designed in a manner similar to that used for the lateral freedom, although the input to the vestibular model 


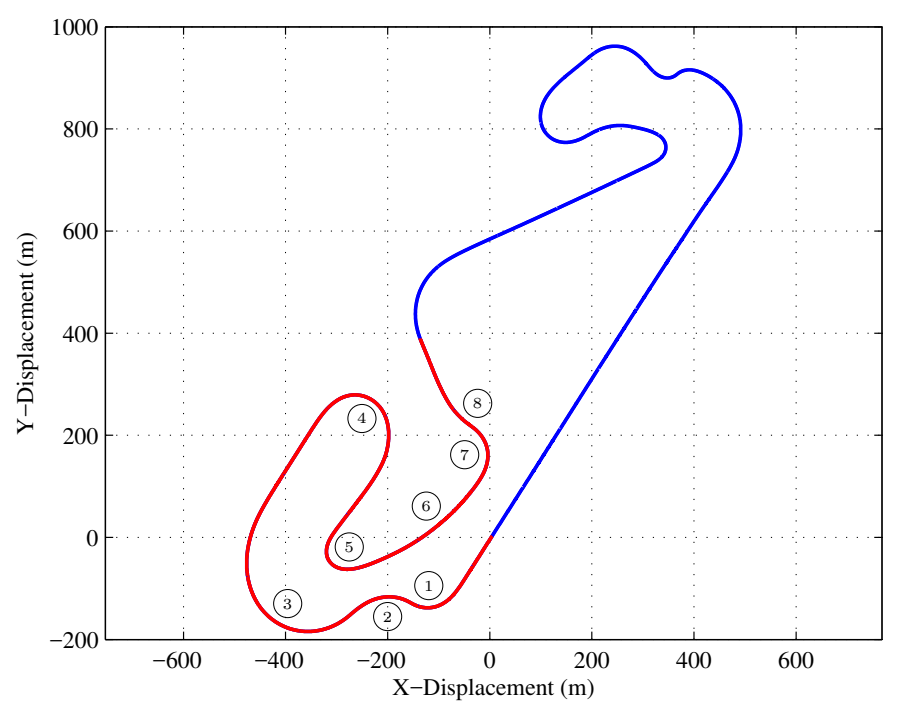

Figure 12. The racing line on the Barcelona Circuit with corner numbers. The cueing filter results are analysed on the section of track highlighted in red.

is yaw velocity.

An alternative yaw cueing strategy is presented in [18], where the vehicle side-slip angle is the platform yaw demand rather than the filtered yaw velocity. There are two advantages associated with using the side-slip angle as a cue: firstly, no filtering (which may remove critical information) is required, and secondly, the driver receives correct vehicle side-slip information. The tests provided in [18] show that in 'normal' driving scenarios side-slip cueing gives better results than traditional yaw-rate cueing. Side-slip angle cueing and filtered yaw-rate cueing will be compared for limit driving in this paper.

\section{Cueing Testing}

Several lateral and yaw cueing strategies were tested on the highlighted portion of the Barcelona closed-circuit track shown in Figure 12. The longitudinal acceleration of the vehicle is shown in the left-hand part of Figure 13. At the beginning of a lap the driver brakes into Turn 1, and then accelerates out of the corner. In Turns 2 and 3, the driver lifts off the throttle on entry into the corner and then accelerates at the exit of the bend. Turns 4, 5 and 7 exhibit a similar decelerate-on-entry and accelerate-on-exit speed profile; the vehicle's lateral and yaw velocities are displayed in the right half of Figure 13. For the most part, these quantities have opposite signs, implying the car's centre of turning is behind the car's centre of mass; see (49). At approximately $20 \mathrm{~s}$ and $27 \mathrm{~s}$ there is a change in direction of the lateral velocity and the centre of turning moves in front of the car's mass centre. In both cases the driver is accelerating out of a corner, and the reduced front-tire loading causes the vehicle to understeer and run wide in the corner, and so additional corrective steering is required.

\subsection{Lateral Filter Tuning}

Several lateral cueing filters were designed by changing the weighting functions $w_{i}(s)$ for the different components of the output $z$ in Figure 11. In previous cueing algorithm studies these weights are constant gains [4], in this work the weights are replaced with first 

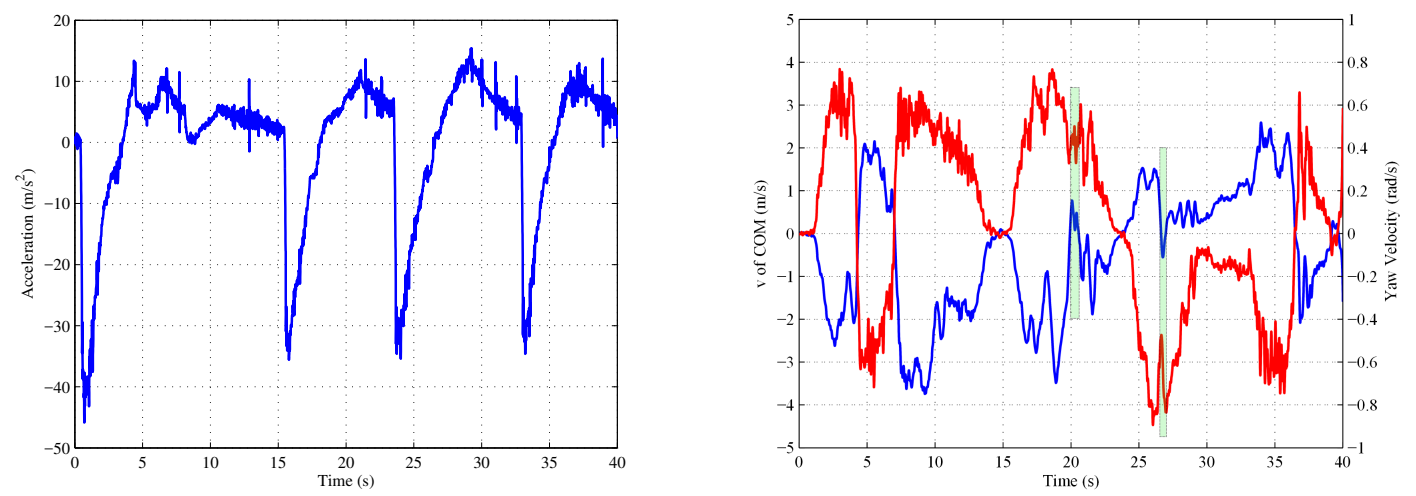

Figure 13. The left-hand plot contains the longitudinal vehicle acceleration for the first eight corners of the Barcelona Circuit. The vehicle's lateral velocity (blue) and the yaw velocity (red) are displayed in the right-hand plot.

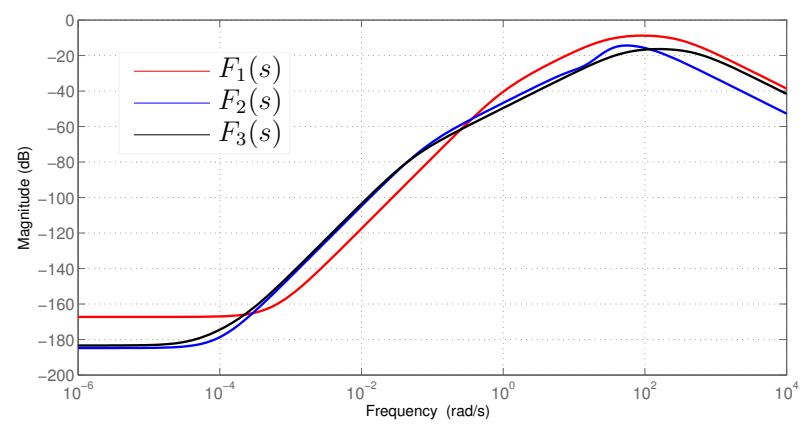

Figure 14. Lateral cueing filters given in (50), (51) and (52).

order filters. This extension provides a lot of additional flexibility in the tuning process. For example, large sustained accelerations that cause platform workspace saturation can be eliminated with frequency shaping that leads to high-pass cueing filters. The three lateral acceleration cueing filters tested here are given by

$$
\begin{gathered}
F_{1}(s)=\frac{117 s^{4}+105 s^{3}+46 s^{2}+0.009 s-1.5 e^{-5}}{s^{5}+324 s^{4}+9355 s^{3}+16935 s^{2}+11355 s+3454} \\
F_{2}(s)=\frac{23 s^{6}+1223 s^{5}+27709 s^{4}+320888 s^{3}+1921608 s^{2}+384 s+0.019}{s^{7}+186 s^{6}+12250 s^{5}+434247 s^{4}+8441725 s^{3}+85856787 s^{2}+419659437 s+32924299} \\
F_{3}(s)=\frac{83 s^{3}+17 s^{2}+0.0033 s-1.7 e^{-7}}{s^{4}+546 s^{3}+25136 s^{2}+6255 s+250} .
\end{gathered}
$$

The Bode magnitude plots for these filters are illustrated in Figure 14, where their highpass properties can be seen.

Figure 15 illustrates the performance of the three filters. The platform acceleration, velocity and displacement for the highlighted section of the Barcelona track shown in Figure 12 are given in plots (a), (b) and (c), respectively. The response of the vehicle lateral acceleration to a perturbation in steering angle, computed in Section 3.1, and shown in Figure 7, are filtered by each of the cueing filter options to determine the 
platform response to a step steering angle input. These are plots are given as plots (d), (e), and (f).

The cueing filter $F_{1}(s)$ is characterised by a strong onset cue as is evident in the platform velocity response, which must be washed out in order to prevent workspace saturation. This cueing option uses the largest 'per-corner' fraction of the workspace (of the three filters) during each corner and returns the platform to a near-central position following each cornering manoeuvre. The discussion in Section 4.1 stressed the importance of cueing behaviours that are qualitatively similar to the vehicle's actual response. In this instance this qualitatively similarity is not retained, because as the vehicle speed increases the platform response to steering perturbations decreases in magnitude as opposed to the opposite trend shown in Figure 7, and no warning of impending instability is provided. When the vehicle is unstable, the cueing filter produces an onset cue, which while longer in duration, is not in the first half second significantly different to the stablecar responses. While this filter was tuned to produce a cueing demand that aims to make full use of the workspace in each corner, and position the platform in the centre in a manner consistent with general cueing wisdom, it loses some critical vehicle behavioural information. Additionally, there is a significant difference between the platform's lateral velocity and the lateral velocity of the car. During the 10-12 s washout period the platform's lateral velocity is in the opposite direction to that of the vehicle. This implies that in the simulator the centre of cornering will move behind the vehicle mass centre in a manner not consistent with the vehicle's actual turning behaviour. The behaviour predicted from this analysis is correlated with the test driver comments who complained of confusing cues that were the opposite of the real vehicle behaviour. He was unable to control the vehicle on the performance limit, which was reflected in increased simulator lap-times. The driver also complained that the platform 'moved too much', suggesting that such strong cues, when accompanied by washout, are undesirable.

The cueing filters $F_{2}(s)$ and $F_{3}(s)$ produce a platform response that is similar to that of the car. The filter $F_{3}(s)$ has a reduced order, because the model of the driver's vestibular system was removed from the design optimisation. In these designs there is no platform washout and consequently little or no false cueing, however, the removal of washout effects enforces the need for low-amplitude cueing. The absence of strong onset cues means that only the high-frequency content of the lateral acceleration is retained in the platform demand signal. At first sight these filters appear flawed - without washout there is no means of preventing workspace saturation. However, in a racing context, where the car is operating on a fixed closed-circuit track, only a limited number of left-hand corners, say, can follow in sequence, which makes the simulator workspace easier to manage. A righthand corner that follows a left-hand corner will 'unwind' the platform and move it back towards the centre of the workspace. For the Barcelona track there are never more than three consecutive left-hand or right-hand corners, and so it is possible to tune the filters to avoid significant platform washout. When the vehicle is unstable the platform cue is large and would normally exceed the workspace. However, with professional drivers, provided the initial cueing is correct, the driver will bring the car promptly under control thereby creating a immediate reduction in the lateral acceleration of the simulator platform. If the driver does lose control, the platform cues no longer matter since the car is likely to have left the track. In the case of the $F_{2}(s)$ and $F_{3}(s)$ filters the platform lateral velocity is in many ways similar to that of the vehicle. Large rapid variations in the platform velocity are no longer present, and the false cues at $20 \mathrm{~s}$ and $27 \mathrm{~s}$ have been removed. Any loss of stability-related information can be partially compensated by yaw cueing strategies that are discussed in more detail in the next section. When the $F_{2}(s)$ and $F_{3}(s)$ filter options were tested, the driver found that the cueing provide realistic 

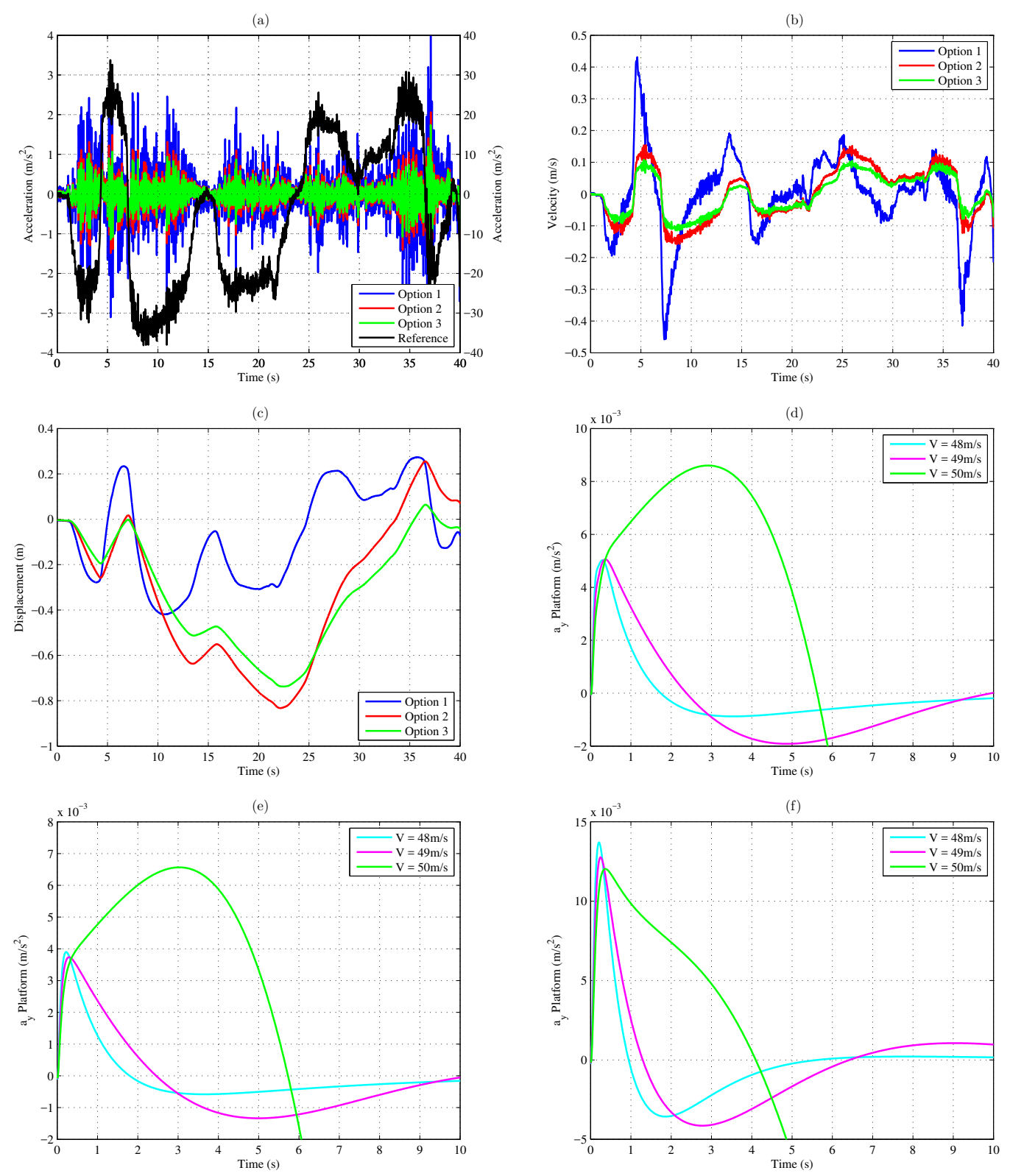

Figure 15. Reference lateral acceleration for the section of the Barcelona circuit highlighted in Figure 12. Figure (a) shows (in black) the vehicles lateral acceleration. The three coloured plots are the simulator lateral acceleration demands for the three $\mathcal{H}_{2}$ optimal filters given in (50), (51) and (52). Figures (b) and (c) show, respectively, the associated platform velocity and displacement demands for each of the three filters. Figure (d) shows the vehicle responses from Figure 7, filtered using $F_{1}(s)$, to determine the platform steering-angle step response at three different vehicle speeds. Figures (e) and (f) contain the same analysis for $F_{2}(s)$ and $F_{3}(s)$.

vehicular information, which helped him to control the oversteer on the entry to corners. The lower-order filter $F_{3}(s)$ was deemed superior, because it has a fast response which delivers timely cues, although they are of lower magnitude. The test driver found that the sooner the cues arrived the easier it was to respond to them and control the vehicle. He also found that $F_{3}(s)$ provided realistic lateral-grip and car 'balance' feedback. 


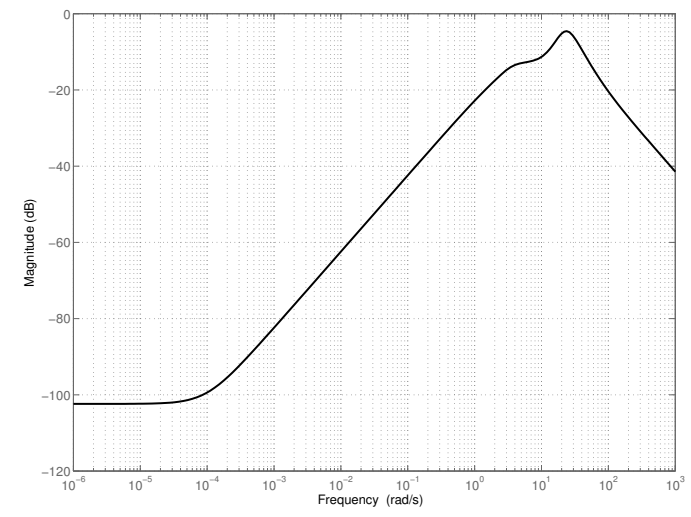

Figure 16. Yaw cueing filter given in (53).
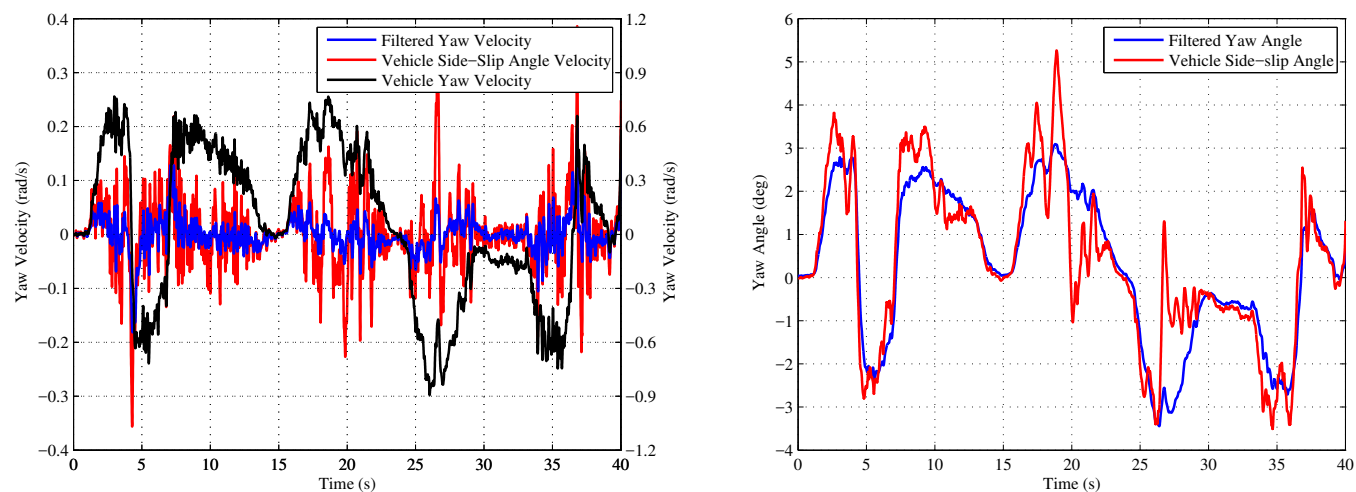

Figure 17. Yaw velocity and side-slip angle cues. The left-hand figure shows the vehicle's yaw velocity (associated with the right axis), the filtered yaw velocity and the vehicle side-slip angle velocity (both associated with the left axis). The right-hand plot shows the platform yaw angle for the filtered yaw velocity and the vehicle side-slip angle.

\subsection{Yaw Filter Testing}

The efficacy of using the side-slip angle as a yaw cue is compared with that of using a more conventional filtered yaw velocity cue. The yaw cueing filter used here was tuned along lines similar to those employed for $F_{2}(s)$ and $F_{3}(s)$ and is given by

$F_{\text {yaw }}(s)=\frac{8.47 s^{6}+635 s^{5}+12012 s^{4}+117627 s^{3}+404881 s^{2}+696129 s+70}{s^{7}+70 s^{6}+2421 s^{5}+49718 s^{4}+590875 s^{3}+2910807 s^{2}+7942101 s+9214671}$,

which has the high-pass Bode magnitude plot illustrated in Fig. 16.

One of the design criteria for the (optimal) yaw filter parameters is that the platform remains within the allowed workspace. For this study the yaw motion is limited to $\pm 4^{\circ}$ and so the cueing signal necessarily contains only high-frequency yaw information. The yaw cue demands for the side-slip angle and filtered yaw angle algorithms are shown in Figure 17. The left-hand plot contains the yaw velocity of the vehicle, the filtered yaw velocity cue and the vehicle side-slip angle velocity. The filtered yaw angle is compared with the vehicle side-slip angle in the right-hand plot.

The two yaw algorithms were tested with the professional driver. During testing the lateral cues were generated using $F_{3}(s)$ in $(52)$ and as shown in Figure 15. With both 
yaw algorithms the driver was able to control the oversteer on entry to the corners, interpret the vehicle behaviour and operate the car on its performance limit. The driver reported that the two options felt 'similar', however, when compared graphically, the cueing signals show similarities as well as differences. As shown in Fig. 17 both cueing options demand similar platform yaw angle swings during corner entry. This is consistent with the driver being able to control the oversteer in corner entry with both algorithms. During corner exit, when the yaw angle is returning to $0^{\circ}$, the cueing demands are almost identical. The differences between the two options occur in mid-corner. In Corner 4 for example, which starts at approximately $15 \mathrm{~s}$, the filtered yaw angle demand increases to $3^{\circ}$ and then decreases to $0^{\circ}$, with only small fractional-degree fluctuations. In contrast, the side-slip angle reached a peak of over $5^{\circ}$ at approximately $18.5 \mathrm{~s}$ when the vehicle understeers and 'runs wide' in the turn. The differences in the platform behaviour during this understeer was not noticed by the driver.

As mentioned in Section 6, and shown in Figure 13, at 20s the lateral velocity of the vehicle rapidly changes sign, which would cause the rotation centre to move infront of the vehicle COM, and would indicate to the driver that vehicle is 'running wide'; this behaviour is not indicated by the lateral cue. As shown in Figure 15 (b), the lateral velocity of the platform remains negative at $20 \mathrm{~s}$; see the $F_{3}(s)$ response. Figure 17 indicates that the yaw velocity of the vehicle remains positive at this time. The yaw velocity cue and the side-slip angle velocity are, however, both negative at $20 \mathrm{~s}$. The platform's centre of turning will therefore move forwards, and a vehicle understeer is cued. This motion is stronger in the side-slip angle case. Referring to Figures 8 and 9, the vehicle yaw velocity response to a steering perturbation is an order of magnitude smaller when the vehicle is accelerating and understeering, as compared with a braking and oversteering vehicle. The lower magnitude angular velocity response will produce a slower change in vehicle position. The driver was thus able to detect understeer from visual cues. The driver also receives feedback through the steering wheel about the vehicle understeer behaviour. There are thus at least three cues contributing to the driver's detecting the vehicle understeer and changes in the motion cues are therefore less noticeable.

\section{Conclusion}

High-performance race car simulators cannot reproduce the accelerations experienced in the actual vehicle and so cueing algorithms are used to filter the vehicle's accelerations retaining only those motions that the driver requires in order to perform specific tasks. Identifying the motion cues that the driver needs is a central problem in cueing algorithm design. The majority of the literature aims to minimise the error in the acceleration sensed by the simulator drivers' vestibular system. In race car simulators the platform's inability to reproduce the large accelerations makes this approach infeasible. Small changes in tuning parameters produce significantly different cueing filters, and without more specific cueing objectives, a lengthy and subjective hit-and-miss testing process is needed.

In racing, the driver attempts to operate the vehicle on its performance limit, and to do so requires timely detailed knowledge of the vehicle handling characteristics. The simulator must cue correctly the vehicle's changing state as it approaches, and possibly over steps, its performance limit. In Sections 2 and 3 the vehicle stability and response to steering perturbations are examined for constant velocity, accelerating and braking situations. An oversteering vehicle becomes progressively unstable as its speed increases. The vehicle also becomes more responsive and changes in its disposition occur quickly. To control the vehicle in this region, the driver requires low-latency motion cues that 
correctly indicate changes in the vehicle's motion. This contrasts with an understeering vehicle that does not go unstable, and has smaller responses to perturbations that change more slowly.

The lateral and yaw cueing filters designed in Section 5 were tuned to cue the changing vehicle behaviour. Importantly, yaw and lateral velocity motions combine to produce a stationary centre of turning, which as shown in Section 4.2 , moves behind the vehicle's mass centre for an oversteering vehicle and in front of it for an understeering one. Any loss of information in the lateral cue can be compensated for by the yaw cue, so that together they produce a turning centre in the simulator that agrees qualitatively with that of the car itself. This approach appears successful as the driver cannot usually identify the cue that provides this information. Indeed, during testing, the yaw cue was changed and the driver was convinced that the lateral cue had been adjusted.

The driver testing of the cueing filters is discussed in Section6. The lateral filters were tuned to capture the vehicle's changing behaviour and provide realistic cues. These cues allowed the driver to control the vehicle at the performance limit; particularly the oversteer at the entry to corners. Both the yaw filter and side-slip angle cueing approaches performed well during testing. The difference between the two options was most noticeable during understeering, but since this is also cued by steering feedback and visual cues this difference was not important to the driver. By studying the vehicle behaviour, and identifying the important motion cues, it is possible to design cueing filters that more nearly meet the race-drivers' requirements. In addition, good cueing results can be found more quickly thereby reducing the test time needed for cue development.

\section{References}

[1] I. Siegler, G. Reymond, A. Kemeny, and A. Berthoz, "Sensorimotor intergration in a driving simulator: contributions of motion cueing in elemtary driving tasks," in Driving Simulation Conference, Sophia Antipolis, France, September 2001.

[2] A. Beghi, M. Bruschetta, and F. Maran, "A real time implementation of MPC based motion cueing strategy for driving simulators," in Proceedings of 51st IEEE Conference on Decision and Control, Maui, Hawaii, USA, 2012.

[3] I. Salisbury and D. Limebeer, "Optimal motion cueing for race cars," IEEE Transactions on Control Systems Technology, pp. 1-14, 2015, DOI: 10.1109/TCST.2015.2424161.

[4] R. Telban, F. Cardullo, and J. Houck, "Developments in human centered cueing algorithms for control of flight simulator motion systems," AIAA Modeling and Simulation Technologies Conference, 1999, Portland. OR. Paper Number AIAA-99-4328.

[5] W. Milliken and D. Milliken, Race car vehicle dynamics. Warendale (PA) SAE, 2005.

[6] H. Radt and D. V. Dis, "Vehicle handling responses using stability derivatives," SAE Technical Paper 960483, 1996.

[7] J. Yi, J. Li, J. Lu, and Z. Liu, "On the stability and agility of aggressive vehicle maneuvers: A pendulum-turn maneuver example," IEEE Transactions on Control Systems Technology, vol. 20(3), pp. 663-676, 2012.

[8] D. Limebeer, R. Sharp, and S. Evangelou, "The stability of motorcycles under acceleration and braking," Journal of Mechanical Engineeering Science, vol. 215(9), pp. 1095-1109, 2001.

[9] A. Tremlett, F. Assadian, D. Purdy, N. Vaughan, A. Moore, and M. Halley, "Quasi-steady-state linearisation of the racing vehicle acceleration envelope: a limited slip differential example," Vehicle Systems Dynamics, vol. 52(11), pp. 1416-1442, 2014.

[10] C. Desoer, "Slowly varying systems $\dot{x}=\mathrm{A}(t) x$, " IEEE Transactions on Automatic Control, vol. 14(12), pp. 780-781, 1969.

[11] H. Rosenbrock, "The stability of linear time-dependent control systems," Journal of Electronic Control, vol. 15(1), pp. 73-80, 1963.

[12] H. Pacejka, Tyre and Vehicle Dynamics. Butterworth-Heinemann Ltd, 2002.

[13] I. G. Salisbury, D. J. N. Limebeer, A. Tremlett, and M. Massaro, "The unification of acceleration 
envelope and driveability concepts," in 24th International Symposium on Dynamics of Vehicles on Road and Tracks (IAVSD), Graz, Austria, 2015.

[14] W. Koiter and H. Pacejka, "Skidding of vehicle due to locked wheels," Proceedings of the Institute of Mechanical Engineers, vol. 183(8), pp. 3-18, 1968.

[15] G. Perantoni and D. Limebeer, "Optimal control of a formula one car with variable parameters," Vehicle Systems Dynamics, vol. 52, no. 5, pp. 653-678, 2014.

[16] J. Slotine and W. Li, Applied Nonlinear Control. Prentice Hall, 1991.

[17] M. Green and D. J. N. Limebeer, Linear Robust Control, ser. Dover Books on Electrical Engineering. Dover Publications Inc., 2012.

[18] N. Garrett and M. Best, "Evaluation of a new body-sideslip-based driving simulator motion cueing algorithm," Proceedings of the Institution of Mechanical Engineers Part D: Journal of Automobile Engineering, vol. 226(11), pp. 1433-1444, 2012.

.




\section{Appendix A. Vehicle and tire Parameters}

The vehicle parameters used in this study are given in Table A1.

Table A1. Formula One car vehicle parameters.

\begin{tabular}{lll}
\hline Symbol & Description & Value \\
\hline $\mathrm{m}$ & Vehicle mass & $720 \mathrm{~kg}$ \\
$\mathrm{~J}$ & Moment of inertia about the z-axis & $450 \mathrm{~kg} \mathrm{~m}^{2}$ \\
$\mathrm{l}$ & Vehicle length & $3.4 \mathrm{~m}$ \\
$\mathrm{a}$ & Distance of mass centre from front axle & $1.8 \mathrm{~m}$ \\
$\mathrm{~b}$ & Distance of mass centre from rear axle & $1.6 \mathrm{~m}$ \\
$\mathrm{~h}$ & Height of mass centre & $0.35 \mathrm{~m}$ \\
\hline
\end{tabular}

The non-linear tire model and parameters used in the this paper are described in detail in [15]. The longitudinal and lateral forces as functions of longitudinal and lateral slip angles are provided for different normal loads.
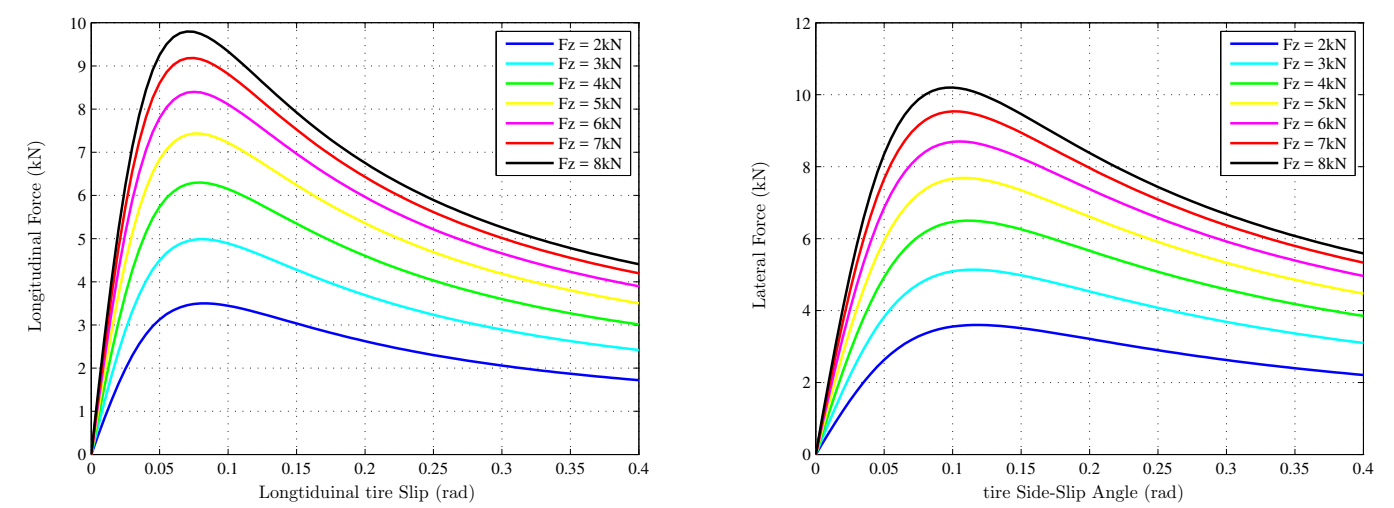

Figure A1. The right-hand figure contains the longitudinal tire force for different normal loads at zero side-slip angle. The left-hand figure contains the lateral tire force for different normal loads at zero longitudinal tire slip. 